\title{
Internal Segregation and Side Chain Ordering in Hairy-Rod Polypeptide Monolayers at the Gas/ Water Interface: An X-Ray Scattering Study
}

\section{Citation}

Fukuto, Masafumi, Ralf K. Heilmann, Peter S. Pershan, Seungju M. Yu, Carissa M. Soto, and David A. Tirrell. 2003. Internal segregation and side chain ordering in hairy-rod polypeptide monolayers at the gas/water interface: An x-ray scattering study. Journal of Chemical Physics 119(12): 6253-6270.

\section{Published Version}

doi:10.1063/1.1602058

\section{Permanent link}

http://nrs.harvard.edu/urn-3:HUL.InstRepos:10356593

\section{Terms of Use}

This article was downloaded from Harvard University's DASH repository, and is made available under the terms and conditions applicable to Other Posted Material, as set forth at http:// nrs.harvard.edu/urn-3:HUL.InstRepos:dash.current.terms-of-use\#LAA

\section{Share Your Story}

The Harvard community has made this article openly available.

Please share how this access benefits you. Submit a story.

Accessibility 


\title{
Internal segregation and side chain ordering in hairy-rod polypeptide monolayers at the gas/water interface: An x-ray scattering study
}

\author{
Masafumi Fukuto, ${ }^{\text {a) }}$ Ralf K. Heilmann,, ${ }^{\text {b) }}$ and Peter S. Pershan \\ Department of Physics and Division of Engineering and Applied Sciences, Harvard University, \\ Cambridge, Massachusetts 02138 \\ Seungju M. Yu \\ Department of Materials Science and Engineering, Johns Hopkins University, Baltimore, Maryland 21218
}

Carissa M. Soto

Naval Research Laboratory, Washington, D.C. 20375

David A. Tirrell

Division of Chemistry and Chemical Engineering, California Institute of Technology, Pasadena, California 91125

(Received 19 May 2003; accepted 27 June 2003)

\begin{abstract}
We report studies of the structure and packing of Langmuir monolayers (LMs) of polypeptide $\operatorname{poly}(\gamma-4-(\mathrm{n}$-hexadecyloxy)benzyl $\alpha, \mathrm{L}$-glutamate) $(\mathrm{C} 16-\mathrm{O}-\mathrm{PBLG})$ on the surface of water. The molecule is a "hairy rod" and consists of side attachments of hexadecyloxy chains (-O-C16) to the rigid rod-like core made up of $\alpha$-helical poly ( $\gamma$-benzyl L-glutamate) (PBLG). Measurements include surface pressure $(\Pi)$ versus area/monomer $(A)$ isotherms, x-ray specular reflectivity $(\mathrm{XR})$, and grazing incidence diffraction (GID). In contrast to the LM of bare PBLG on water, which undergoes a monolayer/bilayer transition with increasing $\Pi$, monolayers of $\mathrm{C} 16-\mathrm{O}-\mathrm{PBLG}$ remain stable up to the highest densities. On the basis of XR and GID results, the structure of the C16-O-PBLG monolayer is characterized by the following main features. First, hydrophobicity causes the $-\mathrm{O}-\mathrm{C} 16$ chains to segregate towards the film/gas interface and away from water and the PBLG cores, which sit parallel to and near the water/film interface. Since the attachment position of some of the side chains is at the core/water interface, the segregation forces these chains into the space between neighboring core rods. Compression associated with increasing $\Pi$ thickens the film but the internally segregated structure is maintained for all $\Pi$ (i.e., $>\sim 30$ dyne $/ \mathrm{cm}$ ). Second, the $\mathrm{C} 16-\mathrm{O}-$ PBLG rods form domains in which the rods are aligned parallel to each other and to the interface. The correlation length for the interhelix positional order of the rods is short and typically comparable to or less than the length of the rods. With increasing $\Pi$ the spacing $d$ between nearest-neighbor rods decreases linearly with $A$ at high $\Pi$, indicating a direct correspondence between the macroscopic compressibility and the microscopic interhelix compressibility. Third, as $\Pi$ increases past $\sim 5$ dyne/cm, the local packing of tethered $-\mathrm{O}-\mathrm{C} 16$ chains displays the same herringbone (HB) order that is common for high-density bulk and monolayer phases of alkyl chains. Various features of the observed GID peaks also imply that the HB order of $-\mathrm{O}-\mathrm{C} 16$ chains is oriented with respect to the helical axes of aligned PBLG cores. We propose that the HB order is established initially by one-dimensionally confined chains between aligned rods at low $\Pi$ and grows laterally with compression. (C) 2003 American Institute of Physics. [DOI: 10.1063/1.1602058]
\end{abstract}

\section{INTRODUCTION}

Rigid $\alpha$-helical polypeptides possessing long side chains are representative of polymers that are often described as "hairy rods." 1,2 The composite character of these molecules implies that they can display structural order at two levels, i.e., in the arrangements of their rigid rod-like cores ("rods") and in the packing of side chains ("hairs"). This is partly responsible for the richness of phase behaviors exhibited by hairy-rod polypeptides in bulk. For example, in the case of poly(glutamate) derivatives with long alkyl side chains,

\footnotetext{
a) Author to whom correspondence should be addressed. Electronic mail: masa@xray.harvard.edu

${ }^{b)}$ Present address: Space Nanotechnology Laboratory, Center for Space Research, Massachusetts Institute of Technology, Cambridge, MA 02139.
}

temperature-dependent studies have revealed crystalline phases with different degrees of side-chain order, a cholesteric liquid crystal that can be induced thermotropically, and an isotropic liquid. ${ }^{3-7}$ The thermotropic liquid crystalline behavior has been attributed to the melting of side chains, which act as a "solvent" for the rod-like cores. ${ }^{3-5}$

Hairy-rod polypeptides can also be assembled in two dimensions (2D) by forming a Langmuir monolayer (LM) at the water/vapor interface. ${ }^{8,9}$ In the LM, the long axes of the molecules are oriented parallel to the monolayer plane, similar to the way in which these rods are oriented in the layered bulk crystals and cholesteric liquid crystals. ${ }^{3-7}$ On the other hand, the LM is not a simple 2D analogue of the layered bulk structures since it is in contact with water on one side of the 
layer. As a result, the structure of the LM is determined by both the "rod-rod" and "hair-hair" interactions as well as by the hydrophobicity of the side chains. Nevertheless, the LMs of hairy-rod polypeptides provide an opportunity to investigate whether the two types of order, namely the rod alignment order and the side-chain packing order, can simultaneously manifest themselves in reduced dimensions. The LM geometry is also advantageous for studying the dependence of these different types of order on the degree of lateral compression.

Over the last decade, Langmuir-Blodgett (LB) films of hairy-rod poly(glutamate)s have attracted considerable interest because of their potential ${ }^{1,2,10}$ for use in optical, ${ }^{11-13}$ optoelectronic, ${ }^{14-16}$ and sensor device applications. ${ }^{17,18} \mathrm{LB}$ multilayer films are most often formed through sequential deposition of a LM on a solid substrate. One of the interesting findings that emerged from the LB studies is that asdeposited LB multilayers often display a bilayer periodicity along the film normal. ${ }^{14-16,19-23}$ The common explanation for this observation is that in the LM state prior to LB deposition, hydrophobic side chains of hairy rods segregate at the film/air interface, resulting in a nonuniform density distribution normal to the monolayer. However, no direct measurements of the corresponding LM structures have been carried out to confirm this inference, albeit a very reasonable one. In the absence of direct structural studies on LMs of hairy-rod polypeptides on water, little is known about the extent to which the various components of these complex molecules are ordered in the LM state.

This paper describes synchrotron x-ray scattering structural studies of LMs formed by the hairy-rod polypeptide $\operatorname{poly}\left(\gamma-4-(\mathrm{n} \text {-hexadecyloxy)benzyl } \alpha \text {,L-glutamate })^{5-7}(\mathrm{C} 16-\right.$ O-PBLG) at various points along surface pressure (П) versus area/monomer $(A)$ isotherms. Figures 1(a) and 1(b) show the chemical structure of the repeating unit of the polymer and a pictorial representation of a hairy rod, respectively. The "rod" part of the molecule C16-O-PBLG consists of the $\alpha$-helical polypeptide $\operatorname{poly}(\gamma$-benzyl L-glutamate $)$ (PBLG), and the "hair" part is given by the hexadecyloxy chains $(-\mathrm{O}-\mathrm{C} 16$; one chain per monomer) that extend out from the PBLG core. The LM of C16-O-PBLG is expected to encompass the structural attributes of two very different types of less complex LM systems whose microscopic structures have already been characterized by previous x-ray scattering studies. The first system of relevance is the LM of bare PBLG. It has been shown recently ${ }^{24}$ that in the monolayer these rod-like PBLG molecules lie down parallel to the interface and align locally with their immediate neighbors. Lateral compression past $\Pi \sim 9$ dyne/cm (at $22{ }^{\circ} \mathrm{C}$ ) results in a first-order monolayer/bilayer transition. In the present study on C16-O-PBLG, we probe the effects that the attachment of extra -O-C16 chains has on the 2D arrangements of PBLG rods and on the stability of the monolayer against formation of a bilayer when external pressure is applied.

Given that the packing of $-\mathrm{O}-\mathrm{C} 16$ chains is another important aspect of the LM structure of C16-O-PBLG, the second type of LM systems of particular relevance are those formed by simple long-chain surfactants, such as fatty acids, alcohols, esters, and phospholipids. ${ }^{25-28}$ The ordered phases

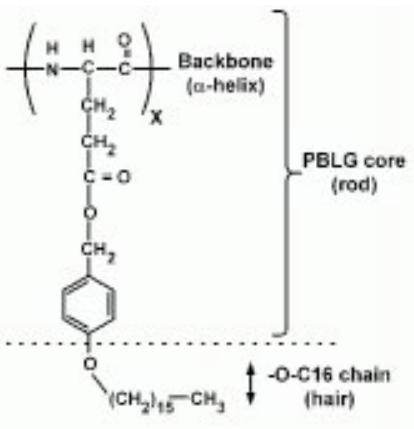

(a)

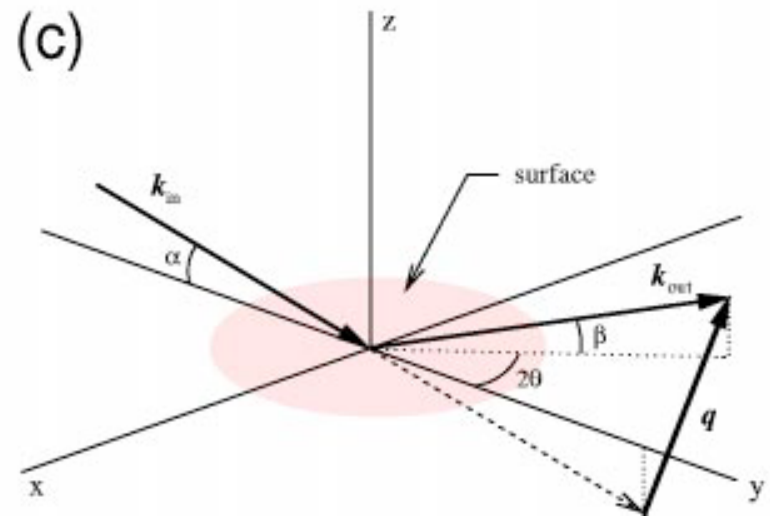

FIG. 1. (a) Chemical structure of the C16-O-PBLG monomer; (b) a pictorial representation of a hairy rod; and (c) x-ray scattering geometry.

of these latter systems have been shown to consist of 2D packing of long alkyl chains that are tilted with respect to the surface normal at low surface pressure $\Pi$ and become untilted at high $\Pi$. In the case of fatty acid LMs, the structural details for each of the several 2D crystalline and liquid crystalline phases (at least eight in total) in the generalized $\Pi-T$ phase diagram are now fairly well established.$^{28}$ In contrast to these LMs of "isolated" alkyl chains, the tethered chains of the C16-O-PBLG LM provide an opportunity to study the $2 \mathrm{D}$ ordering of "constrained" alkyl chains. In particular, one of the principal aims of the present study is to elucidate how the lateral packing of $-\mathrm{O}-\mathrm{C} 16$ chains is influenced by both the tethering to and the ordering of rod-like PBLG cores.

We characterized the LMs of C16-O-PBLG by measuring $\Pi-A$ isotherms at various temperatures and probed the microscopic structure at room temperature by carrying out in situ $\mathrm{x}$-ray specular reflectivity (XR) and grazing incidence diffraction (GID) measurements at various stages of lateral compression. Some of the results have been summarized in a recent short communication, ${ }^{29}$ this paper presents the details of the complete study. The principal results are as follows: (i) The $\Pi-A$ isotherms show that $\mathrm{C} 16-\mathrm{O}-\mathrm{PBLG}$ monolayers sustain high $\Pi\left(>40\right.$ dyne/cm at $\left.22^{\circ} \mathrm{C}\right)$ and that the monolayer/bilayer transition is suppressed in this system. The isotherms for $T>22^{\circ} \mathrm{C}$ exhibit a reversible, relatively narrow plateau-like feature at low $\Pi$ that is absent at $T$ $=11^{\circ} \mathrm{C}$; the origin of this feature will be speculated upon below. (ii) The electron density profiles extracted from the 
XR results are consistent with internal segregation of the monolayer in which the hydrophobic -O-C16 chains are segregated at the film/gas interface and PBLG cores lie parallel to and near the water/film interface. (iii) The GID patterns show a broad peak that originates from local alignment of C16-O-PBLG rods within the monolayer plane. Due to the presence of $-\mathrm{O}-\mathrm{C} 16$ chains that are confined between aligned PBLG cores, the interhelix distance between C16O-PBLG rods is larger than that between bare PBLG rods. (iv) Compression of the film to high surface pressure enhances the lateral packing order of $-\mathrm{O}-\mathrm{C} 16$ chains. Two GID peaks observed at high $\Pi$ are consistent with a common packing mode of alkyl chains, known as the herringbone (HB) packing, ${ }^{28,30,31}$ that is observed in high-density bulk and monolayer phases of alkyl chains. The appearance of only one of the peaks at low $\Pi$ suggests that even when there is no external pressure, the ordered fraction of the $-\mathrm{O}-\mathrm{C} 16$ chains are packed one dimensionally. In order to account for these observations, we propose a model in which the $\mathrm{HB}$ order is established initially by one-dimensionally confined chains between aligned rods and grows laterally with compression. Given that the fatty acid monolayer of comparable chain length would show the HB order at much lower $T\left(<-20{ }^{\circ} \mathrm{C}\right),{ }^{28}$ the room-temperature observation of the $\mathrm{HB}$ order for the $-\mathrm{O}-\mathrm{C} 16$ chains is likely to be a result of reduction in chain entropy due to tethering and confinement.

The rest of the paper is organized as follows: Section II provides a brief description of experimental details. In Sec. III, the experimental results from $\Pi-A$ isotherm, $\mathrm{XR}$, and GID measurements are presented and discussed. In Sec. IV, the main conclusions are summarized.

\section{EXPERIMENTAL DETAILS}

\section{A. Sample and $\Pi-A$ isotherm measurements}

Two different samples of $\operatorname{poly}(\gamma-4$-(n-hexadecyloxy)benzyl $\alpha$,L-glutamate) [C16-O-PBLG; see Fig. 1(a)] molecules were used in this study: a polydisperse, high-MW sample [MW 149000 (vis); DP 325 (vis), PDI 1.32] and a monodisperse, low-MW sample [MW 34 900; DP 76]. Synthesis of the monodisperse sample was accomplished via recombinant DNA methods and has been described elsewhere. ${ }^{7,32}$ Throughout this paper, the polydisperse/ high-MW and the monodisperse/low-MW samples are referred to as "PD325" and "MD76," respectively. Based on the $\alpha$-helix pitch of $L_{1}=1.5 \AA /$ monomer along the helical axis, the length of the rod is about $L=115 \AA$ for the MD76 sample and on the order of $L \sim 500 \AA$ for the PD325 case.

Detailed descriptions of the Langmuir trough, cleaning procedures, temperature control, and film deposition method used have been given previously. ${ }^{33-35}$ The entire trough assembly, including a Wilhelmy balance, is enclosed in a sealed aluminum box. For isotherm measurements, the box was filled with high purity $\mathrm{N}_{2}$ gas. For x-ray measurements, it was filled with high purity $\mathrm{He}$ in order to minimize the background scattering. A Langmuir film of C16-O-PBLG was prepared by spreading a measured volume of chloroform solution on pure water surface (Milli-Q quality). Prior to spreading, the subphase was maintained at the temperature of interest for a period of $1 \mathrm{~h}$ or longer. The nominal concentration of the spreading solutions used ranged from 0.30 to $0.42 \mathrm{mg} / \mathrm{mL}$, and typical volume spread ranged from 90 to $120 \mu \mathrm{L}$. In terms of area/monomer $A$, this corresponded to spreading each film at an initial area of $A>40 \AA^{2} /$ monomer.

For all the isotherm results to be presented, the following stepwise continuous method was used for film compression. Fifteen seconds following each compression step, which was typically $\Delta A \sim 0.25 \AA^{2} /$ monomer, the surface pressure $\Pi$ was measured and the next compression step was taken. The barrier speed used in typical measurements corresponded to a compression rate of $\sim 0.02\left(\AA^{2} /\right.$ monomer $) / s$. During x-ray experiments, the same stepwise continuous method was used for compression from one area of interest to the next, but after the target area/monomer was reached, the film was allowed to relax before measurement.

\section{B. X-ray measurements}

The $\mathrm{x}$-ray experiments were carried out using the Harvard/BNL liquid surface spectrometer ${ }^{33}$ at Beamline $\mathrm{X} 22 \mathrm{~B}$ at the National Synchrotron Light Source, with an x-ray wavelength of $\lambda=1.55 \AA$. The general scattering geometry illustrated in Fig. 1(c) defines scattering angles $(\alpha, \beta$, and $2 \theta)$ and wave vectors. The surface lies in the $x-y$ plane, and the plane of incidence defines the $y-z$ plane $(2 \theta=0)$. The wave vector transfer $\mathbf{q}$ is defined to be the difference between the scattered and incident wave vectors: $\mathbf{q}=\mathbf{k}_{\text {out }}-\mathbf{k}_{\text {in }}$. The components along the surface normal (the $z$ axis) and parallel to the surface plane are given by $q_{z}=(2 \pi / \lambda)[\sin (\alpha)+\sin (\beta)]$ and $q_{x y}=(2 \pi / \lambda)\left[\cos ^{2}(\alpha)\right.$ $\left.+\cos ^{2}(\beta)-2 \cos (\alpha) \cos (\beta) \cos (2 \theta)\right]^{1 / 2}$, respectively. For all measurements, scattered signals were measured by a $\mathrm{NaI}$ scintillation detector. Between the sample and the detector, two sets of crossed Huber slits were placed, one set at $S_{1}$ $=209 \mathrm{~mm}$ after the sample center and the other (detector slits) at $S_{2}=677 \mathrm{~mm}$. In the following, the height and width of slit opening at $S_{i}$ are indicated as $\left(H_{i}, W_{i}\right)$. For small $2 \theta$, the purpose of the slits at $S_{1}$ is to eliminate stray scattering and otherwise reduce background. As discussed below, for large $2 \theta$, the slits at $S_{1}$ determine the $q_{x y}$ resolution.

\section{1. $X$-ray specular reflectivity $(X R)$}

In XR, the fraction $R$ of the incident $\mathrm{x}$-ray intensity that is reflected specularly in the plane of incidence (i.e., $\beta=\alpha$ and $2 \theta=0$, or $\left.q_{x y}=0\right)$ is measured as a function of the incident angle $\alpha$ or wave vector transfer $q_{z}=(4 \pi / \lambda) \sin (\alpha)$. The detector resolutions were defined by the detector slit opening $\left(H_{2}, W_{2}\right)=(2.5 \mathrm{~mm}, 3.0 \mathrm{~mm})$ at $S_{2}$ and corresponded to $\delta q_{x}=0.018 \AA^{-1}, \delta q_{y}=0.00185 q_{z}$, and $\delta q_{z}=0.015 \AA^{-1}$. The specular reflectivity reported here is the difference between this signal at $2 \theta=0$ and the background intensities measured at $2 \theta$ offsets of $\pm 0.25^{\circ}$, corresponding to the full width $\delta q_{x}$ of the detector resolution.

Specular reflectivity originates from the variation in the average electron density profile $\langle\rho(z)\rangle$ across the interface (averaged over appropriate coherence lengths in the $x-y$ plane). For $q_{z} / q_{c}>4-5$, with $q_{c}$ denoting the critical wave 
vector for total reflection $\left(q_{c}=0.0218 \AA^{-1}\right.$ for pure water subphase), the reflectivity $R\left(q_{z}\right)$ from a macroscopically homogeneous surface is well described by the "master formula" approximation ${ }^{36-38}$

$$
\frac{R\left(q_{z}\right)}{R_{F}\left(q_{z}\right)} \cong\left|\int_{-\infty}^{+\infty} d z \frac{d}{d z}\left[\frac{\langle\rho(z)\rangle}{\rho_{\infty}}\right] e^{-i q_{z} z}\right|^{2},
$$

where $\rho_{\infty}$ is the electron density in the bulk subphase ( $\rho_{\infty}$ $=0.334$ electrons $/ \AA^{3}$ for water). $R_{F}\left(q_{z}\right)$ is the Fresnel reflectivity of an ideally flat and sharp subphase/gas interface, which is equal to $R_{F}\left(q_{z}\right)=1$ for $q_{z} \leqslant q_{c}$ (total reflection) and decays algebraically as $R_{F}\left(q_{z}\right) \approx\left(q_{c} / 2 q_{z}\right)^{4}$ for $q_{z} \gg q_{c}$.

\section{Grazing incidence diffraction (GID)}

For this measurement, the incident angle $\alpha$ is fixed below the critical angle $\alpha_{c}$ (at $\lambda=1.55 \AA, \alpha_{c}=0.154^{\circ}$ for water subphase), and scattered intensities are measured as a function of $2 \theta$ or $q_{x y}[\approx(4 \pi / \lambda) \sin (\theta)$ for $\beta \sim 0]$. For $\alpha<\alpha_{c}$, the reflectivity is essentially total, with the only field below the interface being evanescent waves. As a result, diffuse scattering from the bulk subphase is largely suppressed. The existence of 2D order with a repeat distance $d$ along the surface (e.g., a crystalline monolayer) would result in a peak at $q_{x y}$ $=2 \pi / d$. By performing $2 \theta$ (or $q_{x y}$ ) scans near a GID peak at $q_{x y} \sim 2 \pi / d$ but at different heights above the surface horizon (i.e., at various values of $\beta>0$ or $q_{z}>0$ ), one can obtain information about how the $2 \mathrm{D}$ order responsible for the peak is correlated along the surface normal (i.e., the $z$ axis). ${ }^{37}$

The settings used to collect most of the GID data are as follows: The incident angle was fixed at $\alpha=0.12^{\circ}$, and the illuminated footprint on the surface extended over approximately $L_{f} \sim 50 \mathrm{~mm}$ along the beam direction. In view of the fact that the effective width of sample as seen at the detector position increases with $2 \theta$ as $L_{f} \sin (2 \theta)$, the resolution is slightly more complicated than for XR. The slit settings that were used are: $\left(H_{1}, W_{1}\right)=(6.0 \mathrm{~mm}, 3.0 \mathrm{~mm})$ at $S_{1}$ (all cases $),\left(H_{2}, W_{2}\right)=(18.0 \mathrm{~mm}, 2.0 \mathrm{~mm})$ at $S_{2}$ for $2 \theta<\sim 10^{\circ}$, and $\left(H_{2}, W_{2}\right)=(18.0 \mathrm{~mm}, 3.0 \mathrm{~mm})$ for $2 \theta>\sim 10^{\circ}$. The inplane full width at half maximum (FWHM) resolution $\delta q_{x y}$, which depends on $2 \theta, L_{f}$, and $W_{i}$, varied as $\delta q_{x y}$ $\sim 0.070 q_{x y}$ for $0.17 \AA^{-1}<q_{x y}<0.37 \AA^{-1}$. For $q_{x y}>q^{\prime}$ $=0.37 \AA^{-1}$, the resolution was limited by the fixed horizontal opening $W_{1}$ of the front slits and therefore was constant at $\delta q_{x y} \sim 0.026 \AA^{-1}$, but only a fraction $\sim q^{\prime} / q_{x y}$ of the illuminated path $L_{f}$ contributed to the raw intensities observed by the detector. The relatively large vertical opening $\mathrm{H}_{2}$ of the detector slits corresponded to the integration of scattered signals over $\Delta q_{z} \sim 0.11 \AA^{-1}$.

\section{Comments on radiation effects}

At an early stage in the experiment, C16-O-PBLG films were found to be sensitive to $\mathrm{x}$ rays when high incident intensities were used. With a full incident flux $\left(\sim 10^{9} \mathrm{cts} / \mathrm{s}\right)$ striking the film-coated surface, time-dependent changes could be observed in the scattered intensities. In the case of GID, the use of high intensity beams resulted in a loss of diffraction peaks within $1 \mathrm{~h}$. Consequently, both the incident intensity and exposure time were reduced in the subsequent
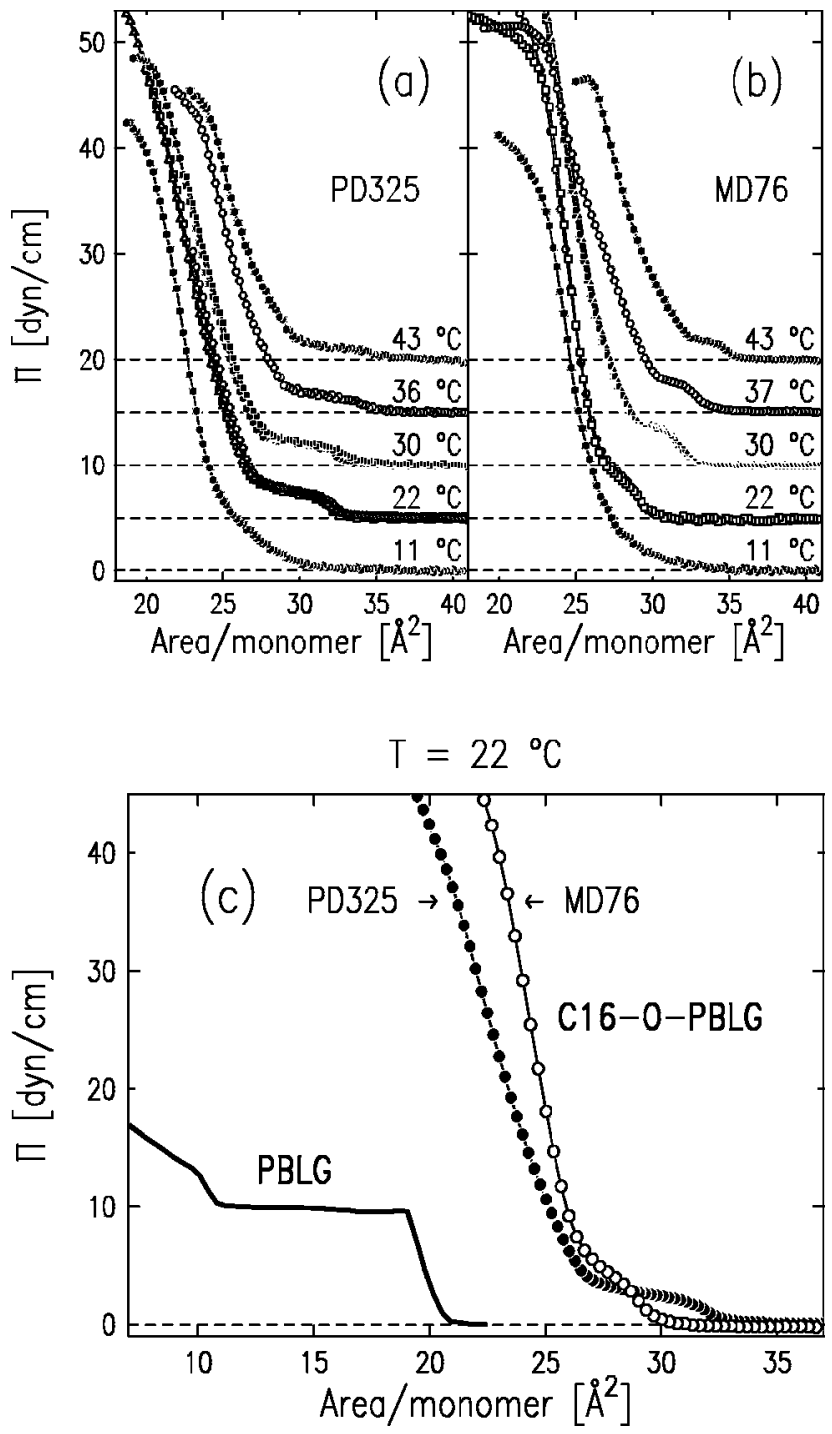

FIG. 2. П-A isotherms from C16-O-PBLG monolayers: (a) PD325 films and (b) MD76 films at various temperatures (shifted vertically for clarity); (c) room-temperature comparison between PD325 and MD76 films of C16-PBLG and bare PBLG.

measurements by using absorbers, small beam divergence, and reduced counting time. Moreover, during measurements at any given $A$, the surface was translated perpendicular to the incident beam [along the $x$ axis in Fig. 1(c)] from time to time to introduce a fresh spot into the beam for illumination. This also served to limit $\mathrm{x}$-ray exposure on any given spot on the film surface. By performing frequent quick reflectivity scans, it was possible to verify that there were no significant radiation-induced changes in the reported film structure. The reduced incident intensity and counting time resulted in some loss of counting statistics in the scattering data; however, there was no other recourse.

\section{RESULTS AND DISCUSSION}

\section{A. ПI- $A$ isotherms}

A series of $\Pi-A$ isotherms measured at various temperatures are summarized in Fig. 2(a) for the PD325 films and in Fig. 2(b) for the MD76 films, respectively. Each curve cor- 
responds to the first compression scan on an as-spread film. For $T=22$ and $30^{\circ} \mathrm{C}$, at which many films were spread, isotherms obtained from separate films are presented together to demonstrate the reproducibility of the isotherms.

Prominent features in the C16-O-PBLG isotherms and their temperature dependence are qualitatively independent of the differences between the two samples. At large areas ( $A>\sim 35 \AA^{2} /$ monomer), the surface pressure remains equal to zero. For $T \geqslant 22^{\circ} \mathrm{C}$, compression past $A \sim 35 \AA^{2} /$ monomer results in a characteristic "shoulder" or plateaulike feature at low surface pressure $(\Pi<\sim 5$ dyne/cm), in which an initial rise in $\Pi$ to a few dyne/cm is followed by a kink and then a much slower increase in $\Pi$. At $T=11^{\circ} \mathrm{C}$, on the other hand, such a shoulder-like feature is absent. In all cases $\left(11 \leqslant T \leqslant 43^{\circ} \mathrm{C}\right)$, further compression above $\sim 5$ dyne/cm results in a steep rise in $\Pi$, which continues until the pressure exceeds a few tens of dyne/cm and the film collapses (indicated by the isotherms bending down at high $\Pi)$. For each of the two samples, the low-П part of the isotherm shifts to larger areas as the temperature is raised, which suggests thermal expansion of C16-O-PBLG films.

In Fig. 2(c), the room-temperature isotherms $\left(22^{\circ} \mathrm{C}\right)$ of the two C16-O-PBLG samples are compared with that of bare PBLG. The PBLG isotherm exhibits a well-defined plateau at $\Pi \sim 9$ dyne/cm, which has been shown to be consistent with a first-order monolayer/bilayer transition. ${ }^{24}$ The very different $\Pi-A$ characteristics displayed by the $\mathrm{C} 16-\mathrm{O}-$ PBLG monolayers must be related to the presence of the additional alkyl chains (-O-C16).

Disregarding the shoulder feature at low $\Pi$ for the moment, extrapolations of the low compressibility part of the isotherms (where $\Pi$ increases sharply) down to the $A$ axis ( $\Pi=0)$ yield $A_{\lim } \sim 27 \AA^{2} /$ monomer for the two samples of C16-O-PBLG at $22^{\circ} \mathrm{C}$. This value of $A_{\text {lim }}$ is clearly greater (by $\sim 30 \%$ ) than $A_{\text {lim }} \sim 20.5 \AA^{2} /$ monomer for PBLG. It should be mentioned that the $A_{\lim }$ value for PBLG is quantitatively consistent with the microscopic structure of the PBLG monolayer, in which the rigid PBLG rods lie down flat on the water surface and are aligned parallel to their immediate neighbors. ${ }^{24}$ The lateral interhelix distance (perpendicular to the helical axis) in an uncompressed PBLG monolayer $(\Pi=0)$ has been shown to be $d=13.6 \AA$. This and the $\alpha$ helix pitch of $L_{1}=1.5 \AA /$ monomer along the helical axis imply a microscopic area/monomer of $A=d \times L_{1}$ $=20.4 \AA^{2} /$ monomer, which agrees with the $A_{\text {lim }}$ estimated from the isotherm. Similarly, the GID results for $d$ versus $\Pi$ to be presented below indicate that the hairy rods $\mathrm{C} 16-\mathrm{O}_{-}$ PBLG are also oriented parallel to the water surface and satisfy the relation $A=d \times L_{1}$ for $\Pi>\sim 5$ dyne/cm. Therefore, the greater value of $A_{\text {lim }}$ for C16-O-PBLG can be interpreted as arising primarily from an increase in interhelix distance between aligned rods.

More quantitatively, the $\sim 30 \%$ difference in $A_{\text {lim }}$ between C16-O-PBLG and PBLG is equivalent to an increase in the interhelix $d$-spacing by $\Delta d \sim 5 \AA$. This difference should correspond to the width of the gap between an adjacent pair of aligned PBLG cores in the C16-O-PBLG monolayer. Note that this width $\Delta d$ is considerably shorter

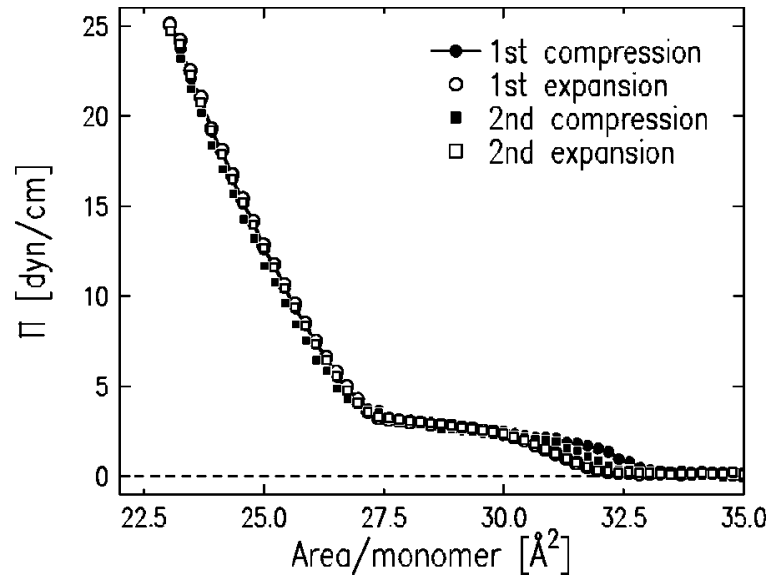

FIG. 3. Compression/expansion isotherms taken on a C16-O-PBLG film (PD325) at $22{ }^{\circ} \mathrm{C}$ (the same film for all scans). The film was compressed only up to $\Pi=25 \mathrm{dyne} / \mathrm{cm}$, and went through two compression/expansion cycles.

than the length of the $-\mathrm{O}-\mathrm{C} 16$ chain, which stretches out to $17 \times 1.27 \AA=21.6 \AA$ in the all-trans conformation. It is however comparable to a typical nearest-neighbor distance $a$ $\sim 5 \AA$ found in the ordered monolayer phases of alkyl chains. ${ }^{28,39}$ According to these considerations, the $-\mathrm{O}-\mathrm{C} 16$ chains that are pinned between a pair of aligned PBLG cores cannot be directed from one core toward the other, parallel to the surface. Instead, it is more likely that such chains point away from the water surface, an inference that is also consistent with their hydrophobicity.

Another important observation is that the C16-OPBLG monolayers withstand relatively high surface pressures, up to $\Pi>40$ dyne $/ \mathrm{cm}$ at $22{ }^{\circ} \mathrm{C}$. This is to be contrasted from the case of the PBLG monolayer, which collapses at $\Pi \sim 9$ dyne/cm to form a bilayer [see Fig. 2(c)]. The origin of the higher stability for C16-O-PBLG monolayers will be illuminated by the $\mathrm{x}$-ray results to be discussed in the following sections.

A unique feature of the C16-O-PBLG isotherms is the appearance of the small shoulder-like plateau at low $\Pi$ for $T \geqslant 22^{\circ} \mathrm{C}$. Evidence that this is not an artifact but originates from compression-induced changes in the internal structure of the monolayer, is provided by the isotherm data that are shown in Figs. 3 and 4. The results of these two different sets of measurements can be summarized as follows:

Figure 3 illustrates the reversibility of the C16-OPBLG isotherms. The four scans shown were obtained from a single film that underwent two compression/expansion cycles at $22{ }^{\circ} \mathrm{C}$. Compression was restricted to $\Pi<25$ dyne $/ \mathrm{cm}$ in order to avoid a significant loss of material from the monolayer that can result from a collapse at high П (e.g., formation of 3D aggregates above or below the monolayer, deposition along the trough edges and the barrier, etc.). It is clear from the overlaps between the first and second compression/expansion scans that the isotherm is reversible as long as the pressure remains well below the collapse point. Moreover, the expansion isotherms consistently exhibit the same shoulder feature, although there is some hysteresis on the large $A$ side where $\Pi \sim 0$. The observation suggests that in the range of $A$ over which this plateau occurs, 


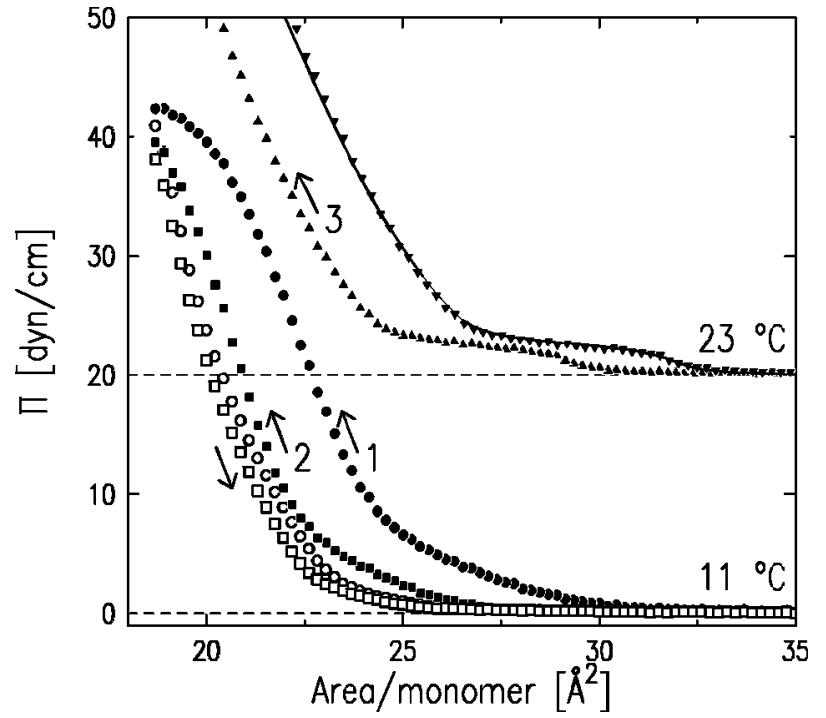

FIG. 4. Compression (filled symbols) and expansion (open symbols) isotherms taken on a PD325 film. The first two cycles were measured at $11^{\circ} \mathrm{C}$. After the second expansion, the film was left undisturbed for $5 \mathrm{~h}$ at $A$ $=42 \AA^{2} /$ monomer while the temperature was raised to $22.7^{\circ} \mathrm{C}$. After a $9 \%$ correction in area/monomer for material loss, the third compression scan (triangles) agrees with an isotherm on a fresh film at $22^{\circ} \mathrm{C}$. The isotherms at $22{ }^{\circ} \mathrm{C}$ have been shifted vertically for clarity.

the surface does not contain bare water areas of macroscopic size. It is conceivable that for a very stiff film, the presence of macroscopic voids might still lead to a small but finite pressure upon compression; however, it seems unlikely that the same feature should appear in the expansion isotherm. If there were macroscopic 2D voids, they should vanish after the first compression and would not reproduce the width of the plateau between the first and second compression isotherms.

The temperature dependence of the shoulder feature, i.e., its presence for $T \geqslant 22^{\circ} \mathrm{C}$ and its absence at $11^{\circ} \mathrm{C}$, is demonstrated more convincingly in Fig. 4. The figure shows another set of isotherms obtained from one film. This film was first subjected to two cycles of compression/expansion at $11^{\circ} \mathrm{C}$, where the compression in each cycle was allowed to go beyond the collapse point. The isotherms shifted to smaller areas due to material loss, which is estimated to be $\sim 8 \%$ after the first compression (from the area shift between the first and second compression scans) and $\sim 1 \%$ after the second compression (from the shift between the first and second expansion scans). Apart from these shifts in $A$, all of these four scans show a monotonic behavior at low $\Pi$. After the second expansion, the film was left undisturbed at $A$ $=42 \AA^{2} /$ monomer for $5 \mathrm{~h}$ while the subphase temperature was raised and stabilized at $22.7^{\circ} \mathrm{C}$. The film was then compressed for the third time but now at $22.7^{\circ} \mathrm{C}$. The corresponding isotherm (up triangles in Fig. 4) now clearly exhibits the shoulder feature at low П. Moreover, when this third scan is shifted horizontally to account for the total material loss of $\sim 9 \%$ due to the first two compressions (down triangles), it agrees very well with the scan on a fresh film at $22{ }^{\circ} \mathrm{C}$ (line).

Some speculations can be made about the origin of the shoulder feature. The fact that this feature is present at room temperature but disappears at low temperature is reminiscent of the coexistence behavior associated with the first-order transition between liquid-expanded $\left(L_{1}\right)$ and liquidcondensed $\left(L_{2}\right)$ monolayer phases of simple surfactant molecules of comparable chain length. The $L_{1}$ phase is a liquidlike phase characterized by disorder in both chain tilt and conformation (i.e., gauche defects), whereas in the $L_{2}$ phase the chains are stretched out and well aligned. ${ }^{25,26,28}$ In the case of fatty acid monolayers, both the $L_{1}$ phase and the compression-induced $L_{1}-L_{2}$ transition are completely suppressed at $T<18{ }^{\circ} \mathrm{C}$ for pentadecanoic acid $(\mathrm{C} 15)^{25,40}$ and at $T<28^{\circ} \mathrm{C}$ for hexadecanoic acid (C16). ${ }^{28,41,42}$ It should also be noted that the bulk melting point of hexadecane (C16) occurs at $18{ }^{\circ} \mathrm{C} .^{39}$ Moreover, the GID results to be presented below show evidence that at room temperature not all but a good fraction of the tethered $-\mathrm{O}-\mathrm{C} 16$ chains are disordered at low $\Pi(<\sim 5$ dyne $/ \mathrm{cm})$. All these considerations suggest that the shoulder feature for $T \geqslant 22^{\circ} \mathrm{C}$ may be somehow associated with chain "freezing" in which some of the conformationally disordered $-\mathrm{O}-\mathrm{C} 16$ chains that are originally present at $\Pi=0$ would be transformed to the all-trans state through lateral compression. According to this interpretation, the chains would already be frozen at $\Pi=0$ for $T=11^{\circ} \mathrm{C}$. On the other hand, one major difference between the observed behavior of the shoulder feature and the $L_{1}-L_{2}$ transition for simple surfactant monolayers is in the dependence on temperature. It is well known that the $L_{1}-L_{2}$ coexistence pressure increases significantly with increasing temperature, e.g., $d \Pi_{L 1-L 2} / d T=1.0-1.2($ dyne $/ \mathrm{cm}) /{ }^{\circ} \mathrm{C}$ for fatty acids. ${ }^{25,40-42}$ The fact that the pressure at the onset of the shoulder feature does not show such strong temperature dependence may be a result of the tethering of $-\mathrm{O}-\mathrm{C} 16$ chains, as opposed to these chains being isolated or "free."

Although the isotherms of the two C16-O-PBLG samples display qualitatively similar overall behaviors, there are quantitative differences between them. The most obvious of all is the difference in the width of the shoulder feature, which is wider for the PD325 sample by a factor of $2-3$. The comparison shown in Fig. 2(c) also indicates that at $22{ }^{\circ} \mathrm{C}$, the PD325 film is more compressible than the MD76 film at high $\Pi$ ( $>\sim 5$ dyne/cm). The question of how these discrepancies between the two samples are related to the differences in dispersity and the molecular size (DP or MW) cannot be answered here. Some of these issues as well as the origin of the shoulder feature are discussed further in the subsequent sections.

\section{B. XR: Segregation within the monolayer}

Representative XR data from C16-O-PBLG films are displayed in Fig. 5 in terms of the normalized reflectivity $R\left(q_{z}\right) / R_{F}\left(q_{z}\right)$. These data sets were obtained from the PD325 films at various stages of compression at $T$ $=22.5^{\circ} \mathrm{C}$, and the corresponding positions along the isotherm are indicated on the right side of the figure. Very similar results have been obtained from the MD76 films at $T$ $=22.5^{\circ} \mathrm{C}$ and $30.9{ }^{\circ} \mathrm{C}$ (not shown). The figure clearly shows that the oscillation periods (in $q_{z}$ ) of the $R / R_{F}$ curves shorten with increasing $\Pi$, which implies thickening of the film as a result of compression. 


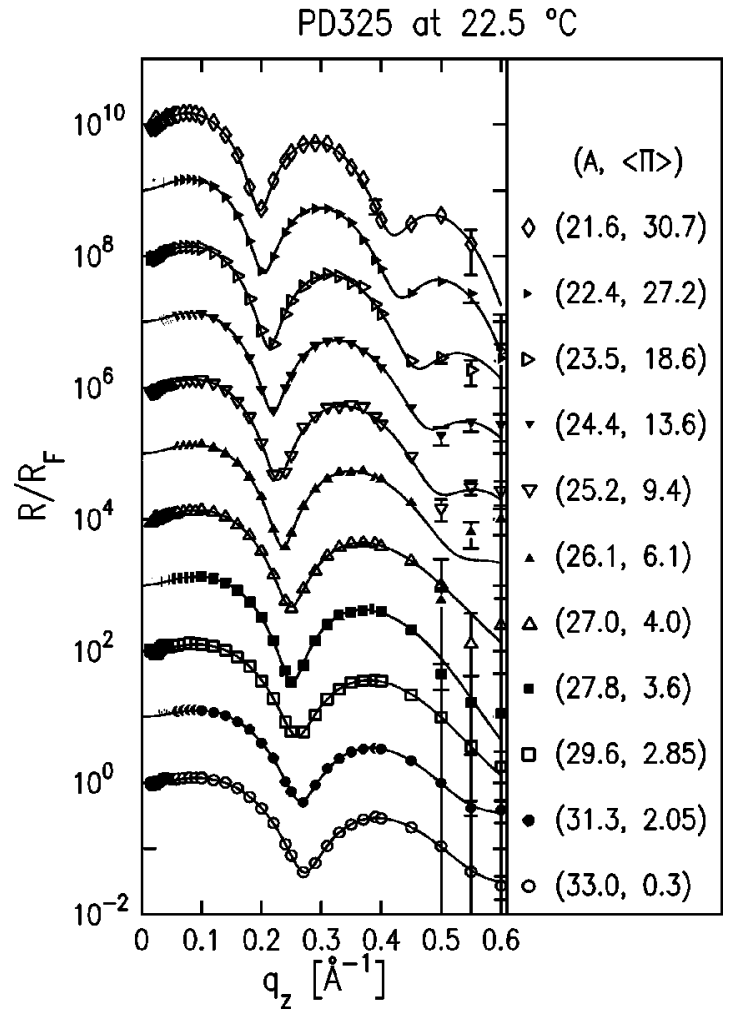

FIG. 5. Specular reflectivity normalized to the Fresnel reflectivity, measured from C16-O-PBLG films (PD325) at $22.5^{\circ} \mathrm{C}$ and at various area/monomer $A$ or surface pressure $\Pi$ (shifted vertically for clarity). The solid lines are the best-fit $R / R_{F}$ curves based on a two-box model for the average electron density profiles $\langle\rho(z)\rangle$.

The extraction of the average electron density profiles across the water/C16-O-PBLG/gas interface has been carried out using a simple " $n$-box" model for $\langle\rho(z)\rangle / \rho_{\infty}$ that is based on the combination of $n+1$ error functions. A schematic representation for the most relevant case of $n=2$ (the "two-box" model) is given in Fig. 6(a). In an $n$-box model, each of the $n$ layers assumed between the water subphase $\left(\rho / \rho_{\infty}=1\right)$ and the vapor above $\left(\rho / \rho_{\infty}=0\right)$ is represented by a box of height $\phi_{i}=\rho_{i} / \rho_{\infty}$ and thickness $l_{i}=z_{i}-z_{i-1}$, and each of the $n+1$ interfaces is then smeared out by a Gaussian roughness $\sigma_{i}$. In the analysis, theoretical $R / R_{F}$ curves based on this model profile and Eq. (1) have been fit to the data in the range $q_{z} \geqslant 0.1 \AA^{-1}$ (i.e., $q_{z} / q_{c} \geqslant 4.5$ ).

For all the reflectivity data obtained from the $\mathrm{C} 16-\mathrm{O}_{-}$ PBLG films, the use of the two-box model with $\phi_{1}>1$ and $\phi_{2}<1$ [see Fig. 6(a)] is both necessary and sufficient to produce good fits. The analysis based on the one-box model $\left(\phi_{i}=0\right.$ for $\left.i \geqslant 2\right)$ leads to fits whose visible discrepancies from the data are too large to be acceptable, indicating that the ability of the two-box model to create a nonuniform density distribution across the film is essential. On the other hand, a relatively small number of measurement points and large uncertainties in the $R / R_{F}$ data at high $q_{z}$ (see the comments on radiation effects in Sec. IIB) would prevent an independent determination of all the two-box parameters if they were allowed to vary freely. Consequently, two kinds of constraints were used in the fitting. First, the thickness of the first layer, which will be later identified as a sublayer domi-
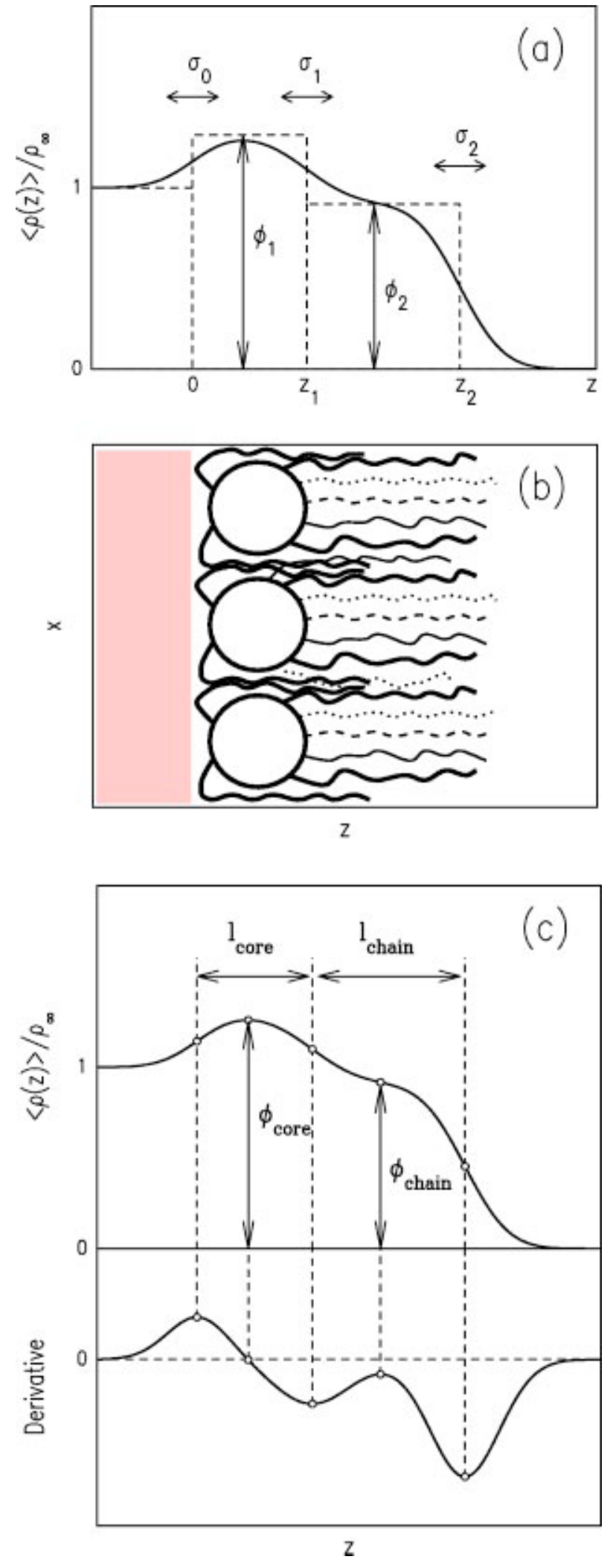

FIG. 6. (a) Two-box model for average electron density profile $\langle\rho(z)\rangle$. The parameters shown in (a) are the fitting parameters that define the profile. (b) Pictorial representation (end-on view) of hairy rods sitting on the water surface. The $-\mathrm{O}-\mathrm{C} 16$ chains prefer to stay away from water and PBLG cores lie parallel to and near the interface with water. (c) New parameters $l_{\text {core }}, l_{\text {chain }}, \phi_{\text {core }}, \phi_{\text {chain }}$ that are derived from the density profiles. They are defined by the extremum points in the profile and the gradient, which is shown at the bottom. 
nated by PBLG cores [see Fig. 6(b)], was constrained as $\mathrm{z}_{1}$ $=l_{1}>9.6 \AA$ on the basis of the smallest observed thickness of $l_{1}=10.6 \pm 1.0 \AA$ for PBLG monolayers. ${ }^{24}$ Second, the lower bound on each roughness parameter $\left(\sigma_{0}, \sigma_{1}\right.$, and $\left.\sigma_{2}\right)$ was set to be equal to the predicted capillary wave roughness $\sigma_{\mathrm{cw}} \cdot{ }^{43,44}$ The latter can be calculated from the surface tension $\gamma=\gamma_{w}-\Pi$ ( $\gamma_{w}$ for water $)$, the relation $\sigma_{\mathrm{cw}}^{2} \propto T / \gamma,{ }^{43,44}$ and the value of $\sigma_{\mathrm{cw}}(\Pi=0)=2.50 \AA$ for a clean water surface at $22.5^{\circ} \mathrm{C}$, which was measured using the same experimental resolutions as for the films. This second constraint neglects any features in the density profile $\langle\rho(z)\rangle / \rho_{\infty}$ that are sharper than $\sigma_{\mathrm{cw}}$.

The best-fit $R / R_{F}$ curves based on the two-box model are indicated by the solid lines in Fig. 5 and the corresponding profiles $\langle\rho(z)\rangle / \rho_{\infty}$ are shown in Fig. 7(a) for the PD325 films. The ranges of the two-box parameters determined from the $\Delta \chi^{2} \sim 1$ contours in the parameter space $(67 \%$ confidence limits) are listed in Table I(a) for three representative films. In Fig. 7(b), comparisons are made between the profiles extracted for PD325 and MD76 films at comparable surface pressures. The bottom two panels in the figure also show the one-box profiles obtained previously for the PBLG monolayer. $^{24}$

Figure 7 shows that for all the C16-O-PBLG films, the lower half of the film (immediately above the water/film interface) is clearly denser than the upper half. While the thickening of the film broadens the profile with increasing $\Pi$, the nonuniform and asymmetric density distribution across the film persists up to high $\Pi$. Figure 7(b) shows that the width of the denser region is comparable to the thickness of PBLG monolayers. As depicted in Fig. 6(b), these observations suggest that the C16-O-PBLG monolayer is composed of the following two physically separate sublayers: (i) a lower layer dominated by the rod-like PBLG cores (i.e., the helical backbone and part of the side chains closer to it) lying parallel to the interface, and (ii) an upper layer consisting primarily of the $-\mathrm{O}-\mathrm{C} 16$ chains. This type of segregation at the submonolayer level is consistent with both the composite character of these hairy rod molecules and the hydrophobicity of the alkyl chains.

The validity of the above interpretation can be checked through quantitative examination of the sublayer thicknesses and densities. Although Table I(a) shows that the uncertainties for the natural parameters used to fit the two-box model (especially for $\phi_{1}, \phi_{2}$, and $\sigma_{1}$ ) are relatively large, the actual density profiles are considerably better defined. This type of situation is typical of models for which the number of independent parameters is larger than can be justified by the data. ${ }^{45}$ In such cases, both the variance of each parameter and the cross correlations between different parameters are large, but linear combinations of these can be found with much smaller uncertainties and cross correlations. In fact, in the present case, more definitive (and more physically meaningful) measures of layer thicknesses and densities can be extracted graphically from the density profiles. We define parameters $l_{\text {core }}, l_{\text {chain }}, l_{\text {total }}\left(=l_{\text {core }}+l_{\text {chain }}\right), \phi_{\text {core }}$, and $\phi_{\text {chain }}$ from the extremum points in the best-fit profiles $\langle\rho(z)\rangle / \rho_{\infty}$ and their gradients, as shown in Fig. 6(c). Table I(b) lists typical ranges of these parameters (derived from the profiles
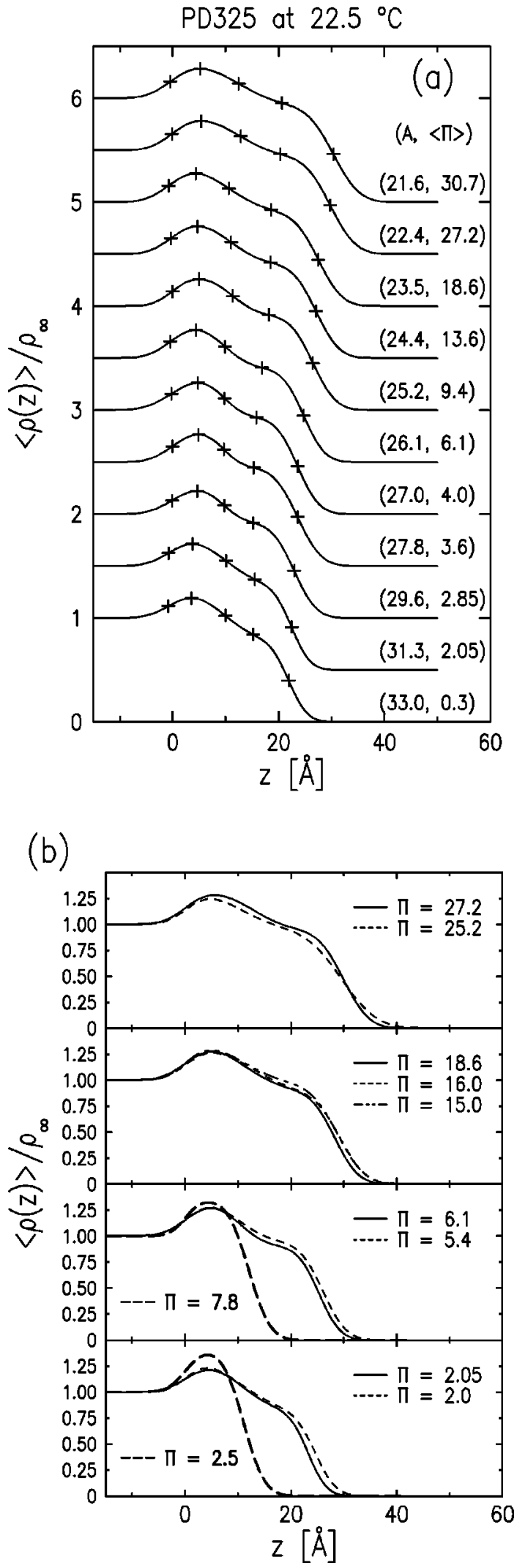

FIG. 7. Average electron density profiles $\langle\rho(z)\rangle / \rho_{\infty}$ of the water/C16-OPBLG/gas interface. They were extracted from the best fits to the $R / R_{F}$ data and are based on the two-box model. (a) PD325 films at various area/ monomer and surface pressures (shifted vertically for clarity). (b) Comparisons between the profiles from PD325 films at $22.5^{\circ} \mathrm{C}$ (solid lines) and MD76 films at $30.9{ }^{\circ} \mathrm{C}$ (short dashed lines) and at $22.5^{\circ} \mathrm{C}$ (long-short dashed lines). The thick and long dashed lines are one-box model profiles from bare PBLG monolayers. 
TABLE I. Typical range of parameters for the average electron density profile $\langle\rho(z)\rangle / \rho_{\infty}$ across the water/ C16-O-PBLG/gas interface, determined by $67 \%$ confidence limits (i.e., from the $\Delta \chi^{2}=1$ contour in the parameter space): (a) two-box fitting parameters; (b) parameters derived from two-box profiles; and (c) comparison between the uncertainties of parameters in (a) and (b). Note: for each parameter $p, \Delta p$ $=$ upper $\operatorname{limit}\{p\}-$ lower $\operatorname{limit}\{p\}$.

\begin{tabular}{|c|c|c|c|c|}
\hline & & $\begin{array}{c}\Pi=2.05 \mathrm{dyne} / \mathrm{cm} \\
\left(\chi_{\min }^{2}=0.81\right)\end{array}$ & $\begin{array}{c}\Pi=9.4 \text { dyne } / \mathrm{cm} \\
\quad\left(\chi_{\min }^{2}=3.3\right)\end{array}$ & $\begin{array}{c}\Pi=30.7 \text { dyne } / \mathrm{cm} \\
\quad\left(\chi_{\min }^{2}=0.75\right)\end{array}$ \\
\hline \multicolumn{5}{|c|}{ (a) } \\
\hline & $z_{1}(\AA)$ & $9.6^{a}-12.7$ & $9.6^{\mathrm{a}}-12.3$ & $9.6^{\mathrm{a}}-14.3$ \\
\hline & $z_{2}(\AA)$ & $22.4-24.1$ & $25.6-27.0$ & $29.5-30.4$ \\
\hline & $\phi_{1}$ & $1.20-1.42$ & $1.25-1.42$ & $1.29-1.59$ \\
\hline & $\phi_{2}$ & $0.72-0.97$ & $0.84-0.96$ & $0.85-0.97$ \\
\hline & $\sigma_{0}(\AA)$ & $2.54^{\mathrm{b}}-3.8$ & $2.68^{\mathrm{b}}-3.9$ & $3.30^{\mathrm{b}}-4.6$ \\
\hline & $\sigma_{1}(\AA ⿻)$ & $2.54^{\mathrm{b}}-7.2$ & $2.68^{\mathrm{b}}-6.2$ & $4.0-9.4$ \\
\hline \multirow{2}{*}{\multicolumn{5}{|c|}{ (b) }} \\
\hline & & & & \\
\hline & $l_{\text {core }}(\AA)$ & $9.7-12.8$ & $10.6-12.3$ & $12.0-14.4$ \\
\hline & $l_{\text {total }}(\AA)$ & $22.8-24.1$ & $26.1-27.0$ & $30.4-31.2$ \\
\hline & $l_{\text {chain }}(\AA)$ & $11.2-13.2$ & $14.7-15.6$ & $16.9-18.4$ \\
\hline & $\phi_{\text {core }}$ & $1.195-1.218$ & $1.247-1.263$ & $1.273-1.288$ \\
\hline & $\phi_{\text {chain }}$ & $0.834-0.961$ & $0.882-0.953$ & $0.937-0.976$ \\
\hline \multicolumn{5}{|c|}{ (c) } \\
\hline & $\Delta l_{\text {core }} / \Delta z_{1}$ & 1.0 & 0.63 & 0.50 \\
\hline & $\Delta l_{\text {total }} / \Delta z_{2}$ & 0.74 & 0.60 & 0.49 \\
\hline & $\Delta \phi_{\text {core }} / \Delta \phi_{1}$ & 0.11 & 0.094 & 0.050 \\
\hline & $\Delta \phi_{\text {chain }} / \Delta \phi_{2}$ & 0.51 & 0.60 & 0.31 \\
\hline
\end{tabular}

a Lower bound on $z_{1}=l_{1}$ constrained by the smallest thickness of $l_{1}=10.6 \pm 1.0 \AA$ observed for PBLG monolayers.

${ }^{\mathrm{b}}$ Lower bound on $\sigma_{i}$ constrained by the predicted capillary-wave roughness.

with $\left.\Delta \chi^{2} \sim 1\right)$, and Table I(c) demonstrates the narrower ranges of their uncertainties by comparing them with those of the corresponding two-box parameters.

The layer thicknesses $l_{\text {core }}, l_{\text {chain }}$, and $l_{\text {total }}$ and the sublayer densities $\phi_{\text {core }}$ and $\phi_{\text {chain }}$ thus derived are plotted as a function of $A$ in Fig. 8 and as a function of $\Pi$ in Fig. 9. The quantity $\rho_{s}$ in Fig. 8(a) represents the number of electrons per unit area belonging to the C16-O-PBLG molecules, which contain $n_{0}=255$ electrons/monomer. The experimentally derived estimates $\rho_{s}=\rho_{\infty}\left(\phi_{\text {core }} \cdot l_{\text {core }}+\phi_{\text {chain }} \cdot l_{\text {chain }}\right)$ (triangles) and the theoretical curve $\rho_{s}=n_{0} / A$ expected for a laterally uniform, monomolecular film (dashed curve) agree within $\sim 10 \%$ of each other, all the way up to $\Pi \sim 30$ dyne/ $\mathrm{cm}$. The good agreement indicates that the two sublayers together account for nearly all of the C16-O-PBLG molecules on the surface. Moreover, it reinforces the validity of the underlying assumption that the C16-O-PBLG film is indeed a monolayer (as opposed to, say, a bilayer) and remains so up to high $\Pi$ with little loss of material.

As already inferred from Fig. 7(b), the thickness $l_{\text {core }}$ of the lower sublayer [squares in Figs. 8(c) and 9(b)] compares well with the thickness $l_{1}=10.6 \sim 12.1 \AA$ observed for PBLG monolayers. $^{24}$ This is consistent with the rod-like PBLG cores of C16-O-PBLG that are oriented parallel to and concentrated near the water/film interface with a relatively narrow height distribution. The plausibility of the maximum density $\phi_{\text {core }}$ can be checked as follows: If the fraction $A_{\mathrm{PBLG}} / A$ of the area in the lower sublayer is attributed to the PBLG cores and the remaining fraction to alkyl chains, upper and lower bounds on $\phi_{\text {core }}$ can be estimated using

$$
\begin{aligned}
& \phi_{\text {core }}^{+}=\left(A_{\mathrm{PBLG}} / A\right) \phi_{\mathrm{PBLG}}+\left(1-A_{\mathrm{PBLG}} / A\right) \phi_{\mathrm{alk}, S}, \\
& \phi_{\mathrm{core}}^{-}=\left(A_{\mathrm{PBLG}} / A\right) \phi_{\mathrm{PBLG}}+\left(1-A_{\mathrm{PBLG}} / A\right) \phi_{\mathrm{alk}, L},
\end{aligned}
$$

where $A_{\mathrm{PBLG}}$ and $\phi_{\mathrm{PBLG}}$ represent the area/monomer and the maximum value of $\langle\rho(z)\rangle / \rho_{\infty}$ for a close packed PBLG monolayer on water, respectively. The quantity $\phi_{\mathrm{alk}, i}$ $=\rho_{\mathrm{alk}, i} / \rho_{\infty}$ stands for the electron density in a crystalline $(i$ $=S)$ or liquid $(i=L)$ phase of alkyl chains normalized to that of water. The parameters for PBLG can be represented by $A_{\mathrm{PBLG}}=20.2 \AA^{2} /$ monomer and $\phi_{\mathrm{PBLG}}=1.36$ observed at $\Pi=2.5$ dyne/cm [see Fig. 7(b)]. As for $\phi_{\text {alk }, i}$, the bulk liquid density of $n$-alkanes ${ }^{39,46}$ can be used to set $\phi_{\text {alk }, L}=0.80$, while electron densities measured previously for ordered monolayer phases of simple surfactants ${ }^{33,36,47-49}$ and for surface frozen and bulk rotator phases of $n$-alkanes ${ }^{39,46}$ lead to $\phi_{\text {alk }, S}=0.95-1.0$. The bounds calculated using these values in Eq. (2) are indicated by the dashed curve $\left(\phi_{\text {core }}^{+}\right.$with $\left.\phi_{\text {alk }, S}=1.0\right)$ and the dotted curve $\left(\phi_{\text {core }}^{-}\right)$in Fig. 8(b). The fact that the experimentally derived values for $\phi_{\text {core }}$ [squares in Figs. 8(b) and 9(a)] fall between these two limiting curves at low $\Pi$ (large $A$ ) provides additional evidence for the segregation of PBLG cores near the water/film interface. On the other hand, the fact that the values for the upper layer density $\phi_{\text {chain }}$ [triangles in Figs. 8(b) and 9(a)] fall within the range $\phi_{\text {alk }, L}=0.80<\phi_{\text {chain }} \leqslant \phi_{\text {alk }, S} \sim 1$ suggests that alkyl chains are the primary constituents of the upper sublayer. This observation is consistent with the segregation of $-\mathrm{O}-\mathrm{C} 16$ chains near the film/vapor interface. 


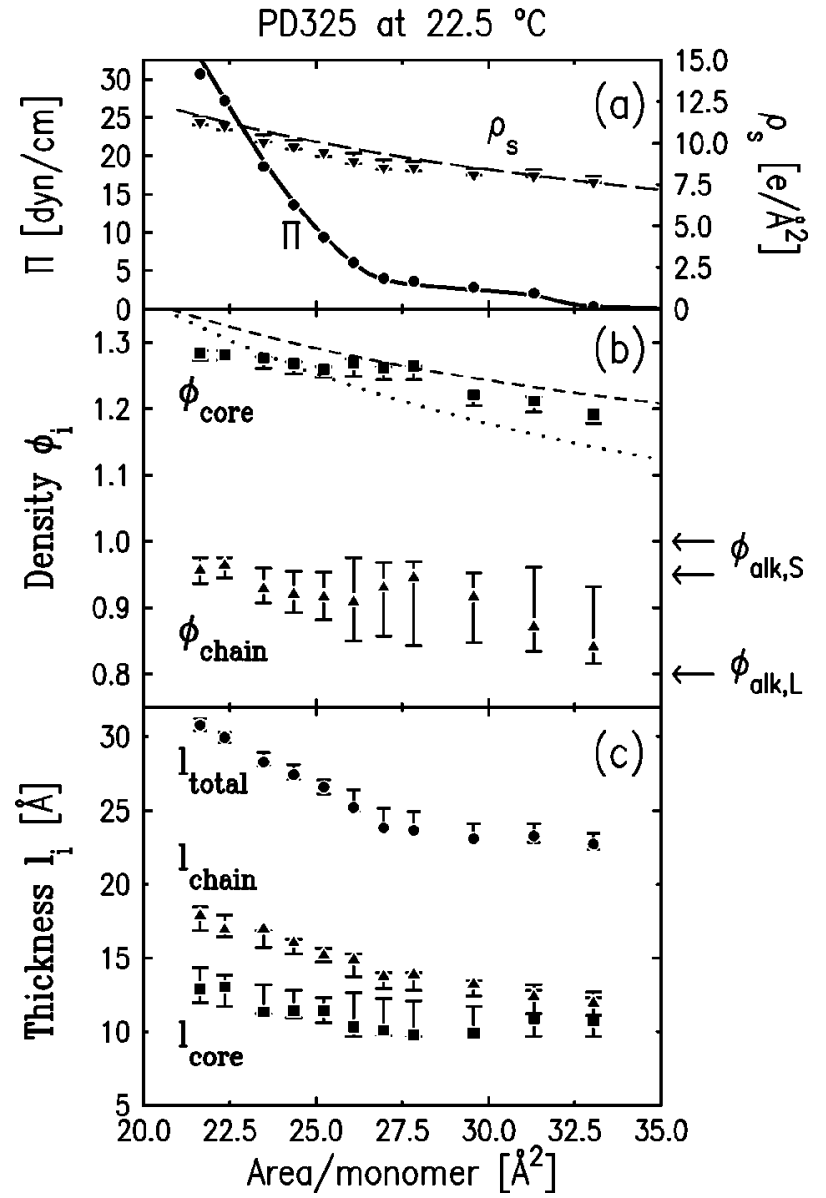

FIG. 8. (a) The surface electron density from C16-O-PBLG molecules, (b) relative density parameters, and (c) thickness parameters derived from bestfit profiles as a function of area/monomer for PD325 film at $22.5^{\circ} \mathrm{C}$. In panel (a), the down triangles correspond to $\rho_{s}=\rho_{\infty}\left(\phi_{\text {core }} \cdot l_{\text {core }}+\phi_{\text {chain }}\right.$ $\left.\cdot l_{\text {chain }}\right)$ based on the extracted parameters, and the dashed line to the theoretical curve $\rho_{s}=n_{0} / A$. In panel (b), the dashed line corresponds to the upper limit $\phi_{\text {core }}^{+}$in Eq. (2a) and the dotted line to the lower limit $\phi_{\text {core }}^{-}$in Eq. (2b). The quantities $\phi_{\text {alk }, i}=\rho_{\text {alk }, i} / \rho_{\infty}$ indicated on the right stand for the electron density in a crystalline $(i=S)$ or liquid $(i=L)$ phase of alkyl chains normalized to that of water.

Figures 8 and 9 show that the behavior of the thicknesses and the densities seem to change around $A_{\text {lim }}$ $\sim 27 \AA^{2} /$ monomer and $\Pi \sim 4$ dyne/cm. The "shoulder" region on the lower density side of this point $\left(A>A_{\text {lim }}\right)$ is characterized by little or no variations in any of the three thicknesses. In this constant thickness regime, the density $\phi_{\text {core }}$ of the lower sublayer starts out (at $\Pi \sim 0$ ) being close to the midpoint between the two limiting curves $\phi_{\text {core }}^{ \pm}$, increases gradually with compression, and becomes comparable to its upper bound $\phi_{\text {core }}^{+}$as the area reaches $A_{\text {lim }}$ $\sim 27 \AA^{2} /$ monomer. Further compression to $A<A_{\lim }$ and $\Pi>5$ dyne/cm produces little further changes in $\phi_{\text {core }}$, but it is now accompanied (together with the steep rise in $\Pi$ ) by film thickening characterized by a slight increase in $l_{\text {core }}$ and more rapid increases in $l_{\text {chain }}$ and $l_{\text {total }}$. These observations (together with $\phi_{\text {core }} \sim \phi_{\text {core }}^{+}$at $A \sim A_{\text {lim }}$ ) suggest that at the limiting area $A_{\text {lim }}$, the lower sublayer achieves the maximum packing configuration that is possible without deformation or vertical displacements of PBLG cores.

The behavior of the upper layer density $\phi_{\text {chain }}$ at low $\Pi$

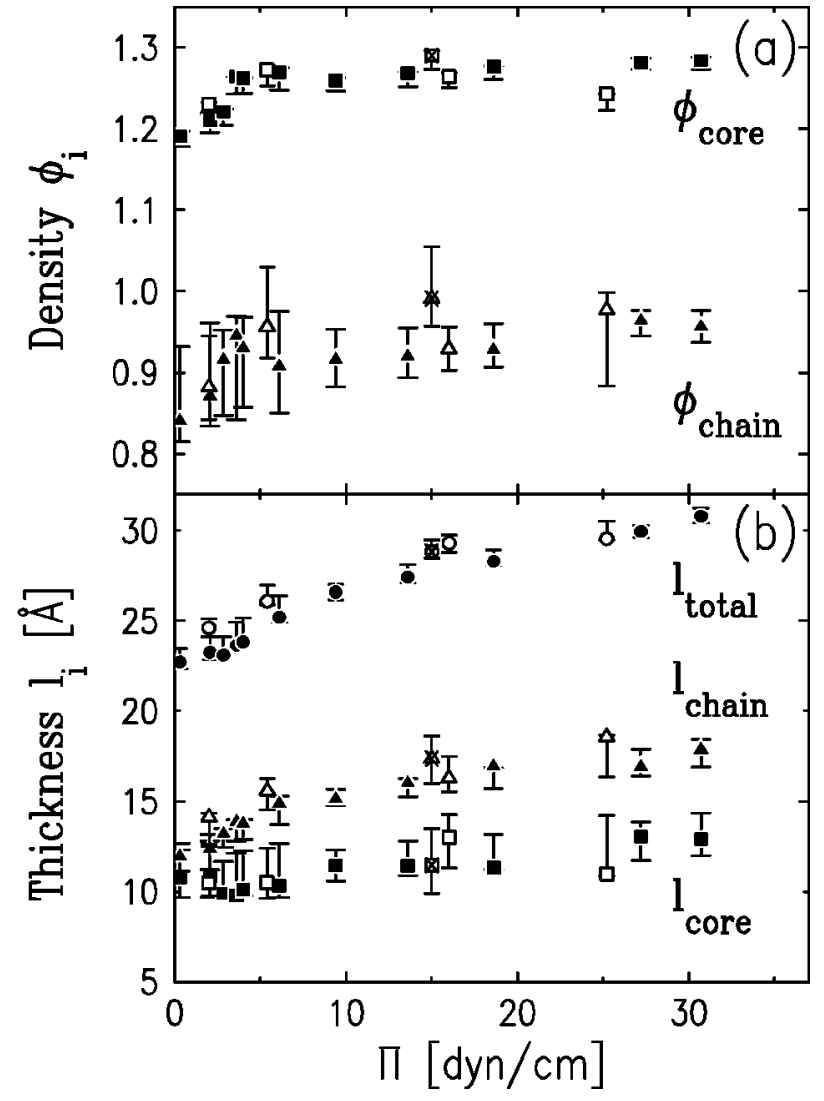

FIG. 9. (a) The relative density parameters and (b) thickness parameters derived from best-fit profiles as a function of $\Pi$; PD325 films at $22.5^{\circ} \mathrm{C}$ (filled symbols); MD76 films at $30.9^{\circ} \mathrm{C}$ (open symbols), and $22.5^{\circ} \mathrm{C}$ (crossed open symbol).

cannot be determined unambiguously due to its relatively large uncertainties. Nevertheless, according to the best-fit values, $\phi_{\text {chain }}$ is closer to $\phi_{\text {alk }, L}=0.80$ at $\Pi \sim 0$ and increases as the area is reduced to $A_{\lim }$. This seems to indicate that at least on the low-density side of the "shoulder" region $(\Pi \leqslant \sim 2$ dyne/cm), the $-\mathrm{O}-\mathrm{C} 16$ chains in the upper layer may be disordered. By contrast, the values for $\phi_{\text {chain }}$ on the high-density side $\left(A<A_{\text {lim }}, \Pi>5\right.$ dyne/cm) are comparable to $\phi_{\text {alk }, S}=0.95-1.0$, suggesting more solid-like packing of these chains. The thickness of $l_{\text {chain }} \sim 18 \AA$ observed at the highest pressure probed $(\Pi \sim 30$ dyne $/ \mathrm{cm})$ would correspond to an average tilt angle of $\theta=\cos ^{-1}(18 / 21.6) \sim 34^{\circ}$ relative to the surface normal for fully extended $-\mathrm{O}-\mathrm{C} 16$ chains if all of them were anchored on a single plane. Since this latter condition is clearly not satisfied in the present case, the above average value should only be viewed as an estimate for possible magnitudes. Given that the side chains are tethered to the helical backbones and some of the alkyl ends reside between PBLG cores in the lower layer, a relatively large distribution in local tilt angles is to be expected. The issues concerning the ordering of $-\mathrm{O}-\mathrm{C} 16$ chains at high $\Pi$ will be illuminated further by the GID results to be discussed in the following section.

\section{GID: In-plane structures}

The GID results reveal that two different types of structural order coexist within the C16-O-PBLG monolayers. 


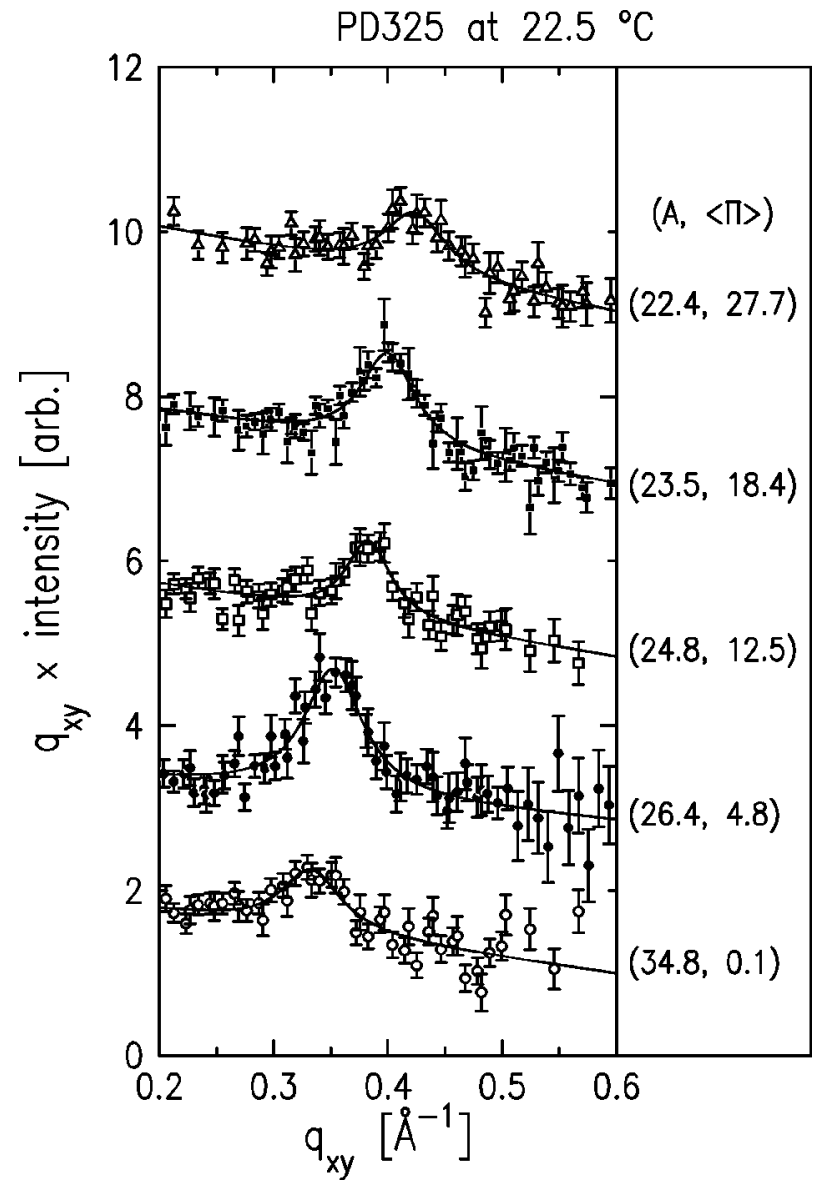

FIG. 10. Interhelix GID peaks from C16-O-PBLG monolayers (PD325) at $22.5^{\circ} \mathrm{C}$ and various $\Pi$. The curves have been shifted vertically for clarity. The lines are Lorentzian fits.

One corresponds to lateral positional order arising from the alignments of rod-like PBLG cores, and the other originates from the packing of chain-like $-\mathrm{O}-\mathrm{C} 16$ part of the side chains. These two types of in-plane order involve dissimilar intermolecular repeat distances and therefore manifest themselves in different ranges of scattering vectors. The principal results elucidating the interrod and interchain structures are presented separately below.

\section{Interhelix order}

GID intensities measured at relatively small lateral wave vectors $q_{x y}$ and near the surface horizon $\left(0<q_{z}\right.$ $<0.11 \AA^{-1}$ ) exhibit a peak centered in the range $0.33<q_{x y}$ $<0.45 \AA^{-1}$. This is illustrated in Fig. 10, which shows a series of representative data collected from PD325 films under different degrees of compression at $22.5^{\circ} \mathrm{C}$. As the film is compressed and the surface pressure rises, the center $q_{0}$ of the peak shifts to larger $q_{x y}$, implying a compressioninduced decrease in the corresponding in-plane repeat distance $d=2 \pi / q_{0}$. The position $q_{0}$ has been determined by fitting a Lorentzian with constant and linear background terms to each GID pattern. The best fits are shown as the solid curves in Fig. 10. The $d$ spacing $d=2 \pi / q_{0}$ is plotted as a function of $A$ in Fig. 11 (PD325 films only) and as a function of $\Pi$ in Fig. 12 (both PD325 and MD76 films), respec-

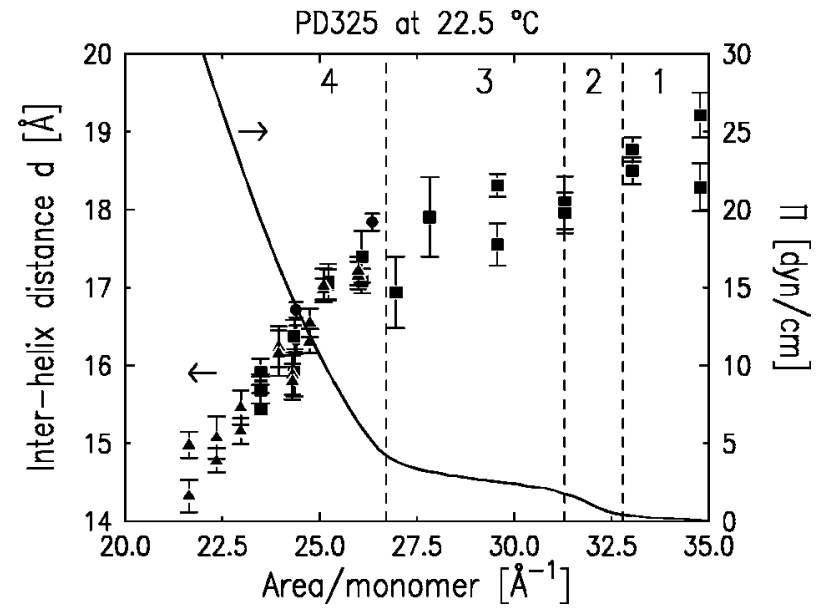

FIG. 11. Interhelix distance (symbols) and surface pressure $\Pi$ (line) as a function of area/monomer $A$ for C16-O-PBLG monolayers (PD325 films) at $22.5^{\circ} \mathrm{C}$. Vertical dashed lines divide the separate regions.

tively. The figures show that the $d$ spacing starts out around $d=19 \AA$ at low $\Pi$ and compression to $\Pi \sim 30$ dyne $/ \mathrm{cm}$ reduces it to $d=14.5 \AA$ for PD325 films and to $d=16 \AA$ for MD76 films.

In the previous GID measurements on PBLG monolayers, a peak that resembles those shown in Fig. 10 was observed but at slightly larger $q_{x y}$ or slightly smaller $d$ spacing. ${ }^{24}$ The similarities with the results on PBLG suggest that the rod-like PBLG cores of C16-O-PBLG molecules are aligned parallel to their near neighbors within the lower sublayer of the monolayer. According to this interpretation, the observed GID peak is associated with the lateral positional periodicity in the direction perpendicular to the aligned rod axes, and the $d$ spacing $d$ can be identified with a nearest-neighbor interhelix distance along the same direction. The main difference between bare PBLG and C16-OPBLG is in the exact magnitude of the interhelix distance. Over the range of $\Pi$ in which the monolayer is stable, the $d$ spacing between PBLG rods has been found to vary from $d=13.6 \AA$ at $\Pi=0$ to $d=12.6 \AA$ at $\Pi=9$ dyne $/ \mathrm{cm} .{ }^{24}$ Figure 12 shows that over the same range of $\Pi$, the interhelix distance between hairy rods $\mathrm{C} 16-\mathrm{O}-\mathrm{PBLG}$ also decreases but

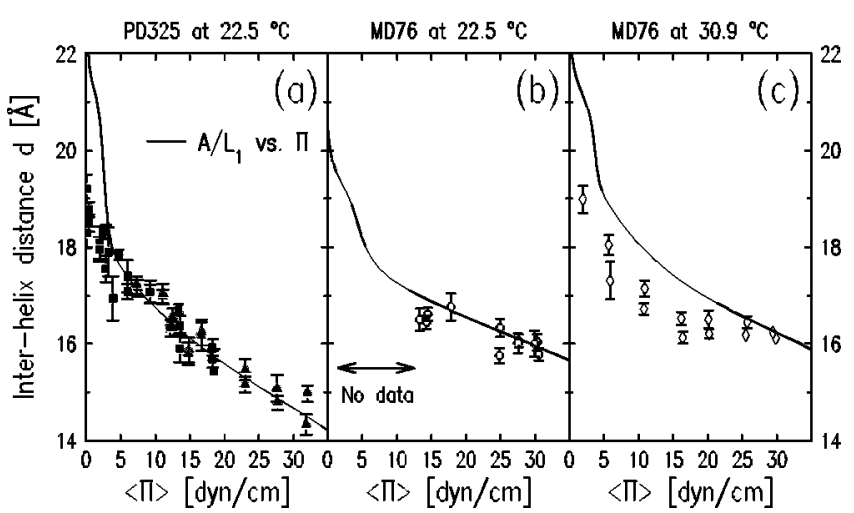

FIG. 12. Interhelix $d$ spacing vs $\Pi$ for C16-O-PBLG monolayers: (a) PD325 films at $22.5^{\circ} \mathrm{C}$, (b) MD76 films at $22.5^{\circ} \mathrm{C}$, and (c) MD76 films at $30.9^{\circ} \mathrm{C}$. Each solid line is given by dividing the area from isotherm by the helical pitch $L_{1}=1.5 \AA$ /monomer along the helical axis. 
remains greater than the values for PBLG by $\Delta d \sim 5 \AA$. This extra $d$ spacing for C16-O-PBLG is consistent with the idea that $-\mathrm{O}-\mathrm{C} 16$ chains are present in the core-dominated lower sublayer and are confined between pairs of aligned PBLG cores.

The widths of the GID peaks observed from C16-OPBLG monolayers do not show any compression-dependent behavior. However, in all cases, the peak widths are broader than the resolution $\delta q_{x y}\left(\sim 0.026 \AA^{-1}\right)$, and the FWHM values $\Delta q_{x y}$ determined from the Lorentzian fits scatter in the range $\Delta q_{x y}=0.04-0.1 \AA^{-1}$. By identifying the inverse of the resolution-corrected half width with a lateral correlation length, ${ }^{28,36}$ i.e., $\xi_{\perp}=2 /\left[\Delta q_{x y}-\delta q_{x y}\right]$, the extent of the lateral positional correlations is estimated to be on the order of only $\xi_{\perp} / d=2-10$ interhelix distances, which implies short-range order. This estimate for $\xi_{\perp} / d$ is comparable to the values found for the PBLG monolayer. ${ }^{24}$ The limited extent of $\xi_{\perp}$ and its apparent insensitivity to changes in $\Pi$ suggest that C16-O-PBLG molecules form a 2D glassy phase consisting of many small, locally ordered domains, which remain "frozen in" up to high П. Moreover, a sample rotation scan with the detector position fixed at the peak center does not show the intensity variations that would be expected if the illuminated region of the sample consisted of few large domains. It follows from this that small domains are oriented randomly within the monolayer plane (i.e., a powder average).

Some insights on how the microscopic structure of the monolayer responds to macroscopic compression can be gained by examining the compression-dependent variations of the interhelix distance. For that purpose, Fig. 11 makes a comparison between the $\Pi-A$ and " $d-A$ " isotherms for PD325 films at $22.5^{\circ} \mathrm{C}$. The figure is divided into four area/ monomer regions (regions 1-4) to emphasize the different surface pressure behavior that can be seen in each region. The behavior of $d$ can be summarized as follows:

In region 1 , in which $\Pi \sim 0$ throughout, the $d$ spacing can be considered, on average, to be roughly constant at $d$ $=18.5-19 \AA$. This is consistent with a macroscopic coexistence of monolayer islands and bare water regions, just as in the case of the PBLG monolayer for $A>A_{\lim }$ and $\Pi=0 .{ }^{24}$ The fact that an interhelix GID peak is already observed in this region indicates a tendency of C16-O-PBLG rods to aggregate laterally and align themselves with neighboring rods immediately upon spreading, without external pressure.

For region 2, the number of data points is too small to make any definite conclusions about a possible change in $d$. Nevertheless, the small initial rise in $\Pi$ in this region appears to be accompanied by a slight decrease in the $d$ spacing to $d \sim 18 \AA$. If this is a real effect of compression, it implies an actual microscopic compression of the C16-O-PBLG monolayer. This suggests that bare water areas of macroscopic sizes are probably absent at the onset of region 2 , as already inferred from the reversibility of the isotherm.

In region 3 , which is defined by the plateau in the $\Pi-A$ isotherm, the $d$ spacing also exhibits a plateau-like behavior. It is interesting to note that the measurable change in surface area over this region $\left(A=31.5 \rightarrow A_{\lim } \sim 27.0 \AA^{2} /\right.$ monomer, or $\Delta A / A \sim 15 \%)$ leaves the interhelix distance almost unaffected (constant at $d \sim 18 \AA$, within a scatter of $\delta d / d$ $\sim 5 \%$ ). Any physical model that attempts to explain the shoulder feature in the isotherm must be able to account for this apparent discrepancy as well.

In region 4, decreasing area results in both a steep rise in $\Pi$ and a significant decrease in the interhelix distance. Therefore, unlike the behavior seen in region 3, macroscopic film compression in region 4 seems to lead directly to microscopic compression of aligned C16-O-PBLG rods within the locally ordered domains.

The relationship between macro- and microscopic compression is illuminated further by the $d$ versus $\Pi$ plot shown in Fig. 12. Each of the " $A / L_{1}$ versus $\Pi$ " curves shown (solid lines) has been obtained by dividing the nominal area/ monomer $A$ in the isotherm by the $\alpha$-helix pitch of $L_{1}$ $=1.5 \AA /$ monomer. These curves are based on the assumption that the total length $L$ of a C16-O-PBLG rod consisting of $n$ monomers is fixed at $L=n \times L_{1}$ at all times. Figure 12(a) shows that for PD325 films at $22.5^{\circ} \mathrm{C}$, the observed $d$ spacing is described very well by the linear relation $d=A / L_{1}$ for $\Pi>\sim 5$ dyne/cm (i.e., in region 4). Although the data are less complete for MD76 films, Figs. 12(b) and 12(c) show that the behavior $d=A / L_{1}$ also seems to hold for the MD76 films at high $\Pi\left(\geqslant 15\right.$ dyne $/ \mathrm{cm}$ at $22.5^{\circ} \mathrm{C}$ and $\geqslant 25$ dyne $/ \mathrm{cm}$ at $30.9^{\circ} \mathrm{C}$ ). These observations imply that at high $\Pi$, close packing of ordered domains almost completely accounts for the entire surface coverage, and there is a direct correspondence between intermolecular and macroscopic compressions.

By contrast, Fig. 12(a) shows that for PD325 films at low $\Pi(<\sim 5$ dyne/cm), the observed $d$ spacing clearly deviates from the $A / L_{1}$ curve but simply extrapolates the high- $\Pi$ behavior down to $\Pi=0$. This deviation is equivalent to the discrepancy noted earlier between the magnitudes of variations in $d$ and $A$ across region 3 (see Fig. 11). For the MD76 film at $30.9^{\circ} \mathrm{C}$, a slight difference between $d$ and $A / L_{1}$ $(>d)$ seems to persist up to a pressure of $\Pi \sim 20$ dyne $/ \mathrm{cm}$, which is well above the values along the shoulder feature. These results indicate that a compression mode other than the reduction of interhelix distance is operating at low $\Pi$.

One possible explanation for the observation $A / L_{1}>d$ at low $\Pi$ is that in the region of isotherm showing the "shoulder" feature, the film-coated surface is still not homogeneous at the microscopic level. The behavior $d=A / L_{1}$ in region 4 and the relatively narrow width of the shoulder feature $(\Delta A / A \sim 15 \%$ for PD325) suggest that even in region 3, a substantial fraction of the surface is occupied by the locally ordered domains. The remaining fraction of the surface area may be due to microscopic holes within such a domain and/or small gaps where neighboring domains meet. Another plausible possibility is the presence of lower-density domains on the surface in which the rods are not perfectly aligned and therefore occupy, on average, a larger area/ monomer than $A_{\text {lim }} \sim 27 \AA$ /monomer. This type of disorder might occur near the boundaries of ordered domains. According to these hypotheses, the compression across region 3 would be accomplished by reducing microscopic areas that are either empty or disordered, while keeping the intermolecular spacing in ordered domains more or less intact. The GID results presented here cannot distinguish between these 


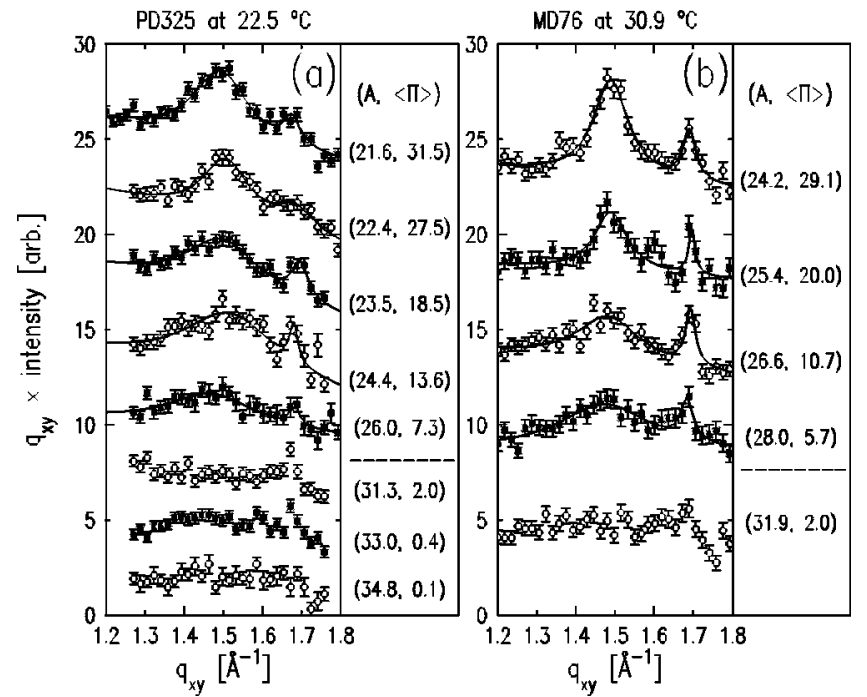

FIG. 13. High- $q_{x y}$ GID scans on C16-O-PBLG monolayers at various surface pressures $\Pi$ : (a) PD325 films at $22.5^{\circ} \mathrm{C}$ and (b) MD76 films at $30.9^{\circ} \mathrm{C}$. The curves have been shifted vertically for clarity.

possibilities. In Sec. III A, it was speculated that the shoulder feature in the isotherm may be associated with freezing of some of the $-\mathrm{O}-\mathrm{C} 16$ chains that are conformationally disordered at low $\Pi$. This would be qualitatively consistent with the above microscopic models of compression if the disordered chains to be frozen corresponded to those that are located at the peripheries of locally aligned domains, at microscopic defects within such domains, or in unaligned, low density regions.

Finally, as far as the interhelix order is concerned, the most prominent difference between the two samples of $\mathrm{C} 16-$ O-PBLG monolayers appears to be in the interhelix compressibility. The discrepancy between the slopes of their $\Pi-A$ isotherms has already been emphasized in Fig. 2(c). Similarly, comparison between Figs. 12(a) and 12(b) shows that locally ordered domains formed within the MD76 film at $22.5^{\circ} \mathrm{C}$ are less compressible than those in the PD325 film. Since both of these films display the behavior $d=A / L_{1}$ at high $\Pi$, it is clear that the apparent discrepancy between their macroscopic compressibilities arises directly from the difference in interhelix compressibility. It is unclear how this effect depends on the differences in sample dispersity and/or molecular size between the two samples. However, there is further evidence that the effect is also related to the extent to which the $-\mathrm{O}-\mathrm{C} 16$ chains are ordered, which will be discussed in the next section.

\section{Side chain order: Confinement-induced herringbone (HB) order of tethered alkyl chains}

The results of GID measurements at large $q_{x y}$ $\left(>1 \AA^{-1}\right)$ show evidence for lateral ordering of $-\mathrm{O}-\mathrm{C} 16$ chains in the C16-O-PBLG monolayer. Figure 13 illustrates typical GID scans near the surface plane $\left(0<q_{z}\right.$ $<0.11 \AA^{-1}$ ) over the range of $q_{x y}$ where the lowest-order peaks due to packing of alkyl chains are observed. In the figure, data collected at various stages of compression are shown separately for PD325 films at $22.5^{\circ} \mathrm{C}$ and a MD76
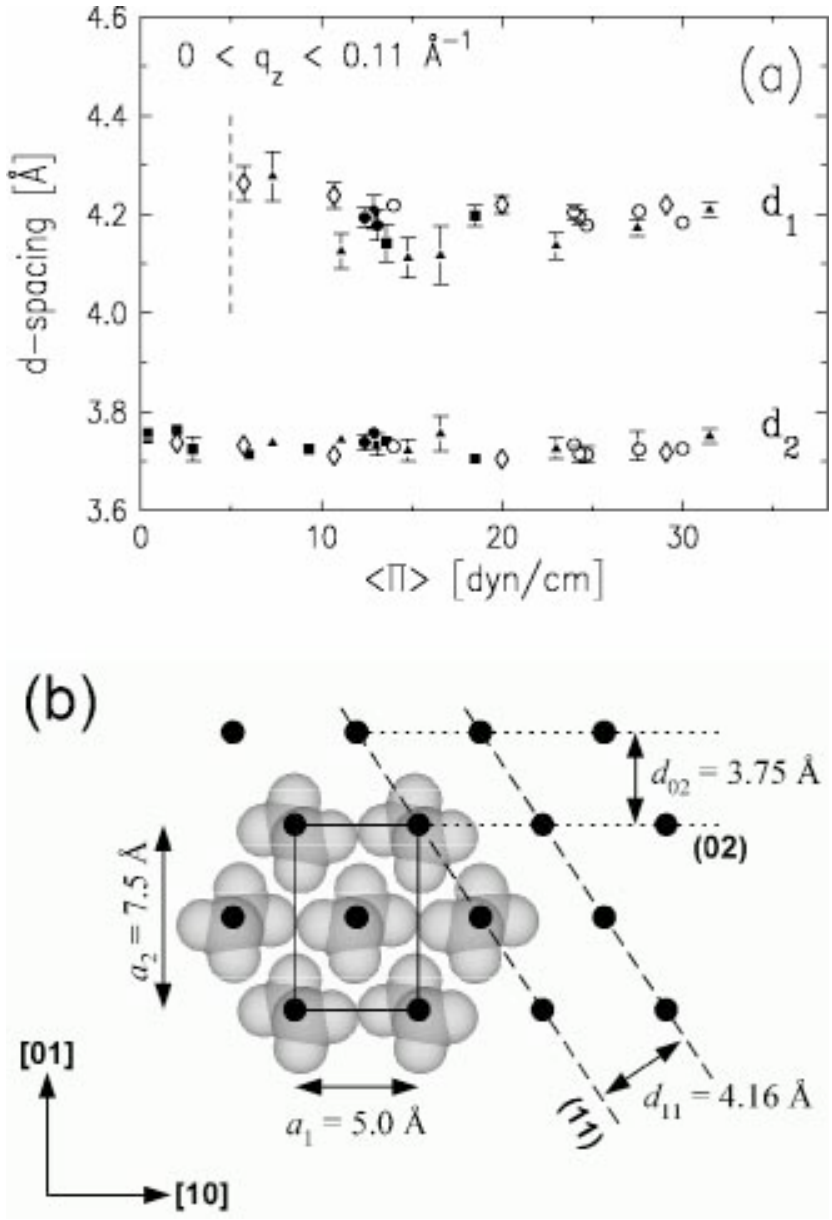

FIG. 14. (a) The $d$ spacing $d_{1}=2 \pi / q_{1}$ and $d_{2}=2 \pi / q_{2}$ extracted from the GID measurements on C16-O-PBLG monolayers: PD325 films at $22.5^{\circ} \mathrm{C}$ (filled symbols) and MD76 films at $22.5^{\circ} \mathrm{C}$ (open circles) and at $30.9^{\circ} \mathrm{C}$ (open diamonds). (b) End-on view of the HB packing arrangement of alkyl chains and the rectangular $\mathrm{HB}$ unit cell.

film at $30.9^{\circ} \mathrm{C}$. Salient features in the GID patterns are independent of the differences between the two samples and can be summarized as follows: First, a relatively broad peak centered at $q_{x y}=q_{1} \sim 1.5 \AA^{-1}$ appears at high $\Pi(>\sim 5$ dyne/ $\mathrm{cm})$. This "first peak" is present only on the high-density side of the shoulder feature in the isotherm and seems to grow with increasing $\Pi$. Second, another peak that is weak but often sharper than the other peak is observed at $q_{x y}$ $=q_{2} \sim 1.68 \AA^{-1}$. This "second peak" seems to be always present, even at low $\Pi$ where the isotherms exhibit the plateau-like behavior. In contrast to the interhelix peak observed at smaller $q_{x y}$, these two peaks shift very little with varying $\Pi$. Qualitatively, these observations imply that compression increases the number of $-\mathrm{O}-\mathrm{C} 16$ chains belonging to an ordered packing structure while leaving the interchain spacings in such a structure almost unaffected.

The observed GID patterns can be fitted fairly well by one or two Lorentzians with constant and linear background terms. The best fits to high- $\Pi$ data containing the two peaks are indicated by the solid curves in Fig. 13. The extracted $d$ spacings $d_{i}=2 \pi / q_{i}$ are plotted as a function of $\Pi$ in Fig. 14(a). The two $d$ spacings are nearly independent of $\Pi$ and can be considered constant at $d_{1} \sim 4.2 \AA$ and $d_{2} \sim 3.75 \AA$. 

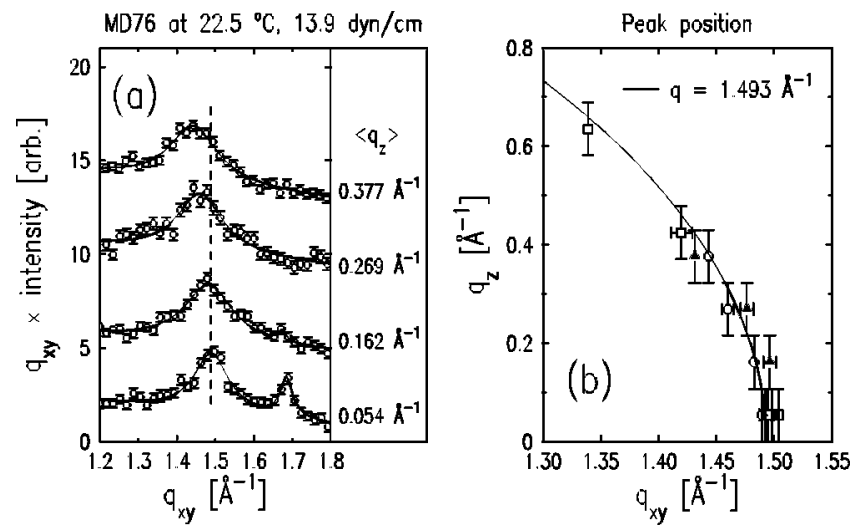

FIG. 15. (a) $q_{x y}$ scans at various height $\left\langle q_{z}\right\rangle$ above the surface (vertically shifted for clarity) measured on a MD76 film at $22.5^{\circ} \mathrm{C}$ and $\Pi=13.9$ dyne/ $\mathrm{cm}$. (b) Peak positions in reciprocal space: MD76 films at 13.9 dyne $/ \mathrm{cm}$ (open circles) and at 24.0 dyne/cm (open squares), and a PD325 film at 31.3 dyne/cm (filled triangles), all at $22.5^{\circ} \mathrm{C}$.

The lack of data for $d_{1}$ below $\sim 5$ dyne/cm signifies the absence of the first peak at low $\Pi$.

The $q_{z}$ dependence of the GID peaks has been probed by means of a series of $q_{x y}$ scans at various detector heights $\left\langle q_{z}\right\rangle$ above the surface plane. The measurements of this type were restricted to films at high $\Pi$, for which the first peak at $q_{x y}=q_{1}$ is well pronounced. Representative data are shown in Fig. 15(a) for a MD76 film at $\Pi=13.9$ dyne $/ \mathrm{cm}$ and $T$ $=22.5^{\circ} \mathrm{C}$. All available data for the positions $\left(q_{x y}\right.$ $\left.=q_{1},\left\langle q_{z}\right\rangle\right)$ of the first peak are presented together as a reciprocal space plot in Fig. 15(b), where the vertical error bars for $q_{z}$ represent the fixed vertical detector acceptance $\Delta q_{z}$ $=0.11 \AA^{-1}$. The results show that with increasing $q_{z}$, the first peak at $q_{x y}=q_{1}\left(\sim 1.5 \AA^{-1}\right.$ at $\left.q_{z} \sim 0\right)$ continually shifts to smaller $q_{x y}$ while its magnitude changes little. Up to the largest $q_{z}$ value of $\left\langle q_{z}\right\rangle_{\max } \sim 0.64 \AA^{-1}$ (close to the experimentally accessible limit), the shift in the peak center roughly follows a circle of constant $q \sim 1.5 \AA^{-1}$ [solid curve in Fig. 15(b)], where $q^{2}=q_{x y}^{2}+q_{z}^{2}$. This behavior of the first peak is indicative of a range of tilts for the orientations of $-\mathrm{O}-\mathrm{C} 16$ chain axes relative to the surface normal, as discussed further below.

By contrast, the second peak at $q_{x y}=q_{2} \sim 1.68 \AA^{-1}$ is centered at $q_{z}=0$, diminishes rapidly with increasing $q_{z}$, and disappears almost completely for $\left\langle q_{z}\right\rangle>0.2 \AA^{-1}$. A quantitative characterization of the intensity falloff along $q_{z}$ is hindered by a combination of the use of relatively wide acceptance $\Delta q_{z}$, the nevertheless small magnitude of this peak, and a background due to the tail of the first peak. However, some estimates can be made on possible forms of this falloff. For example, suppose that vertical (or "untilted") straight chains of length $l$ were to form a monolayer with no distribution in their heights $h$ (i.e., $\left\langle h^{2}\right\rangle=0$ ). Then the intensity distribution along a Bragg rod would be described by the form $S_{\text {rod }}\left(q_{z}\right)=T_{F}(\beta)\left[\sin \left(q_{z} l / 2\right) /\left(q_{z} l / 2\right)\right]^{2}$, where $T_{F}(\beta)$ is the Fresnel transmission factor of an ideally flat and sharp water/vapor interface as a function of the output angle $\beta .^{33}$ The intensity $I_{\text {rod }}\left(\left\langle q_{z}\right\rangle\right)$ actually observed is equal to the integration of $S_{\text {rod }}\left(q_{z}\right)$ over $\left|q_{z}-\left\langle q_{z}\right\rangle\right| \leqslant \Delta q_{z} / 2$. Using $l$ $=21.6 \AA$ for the all-trans length of $-\mathrm{O}-\mathrm{C} 16$, one would ex- pect the ratio $I\left(\left\langle q_{z}\right\rangle\right) / I\left(0.054 \AA^{-1}\right)$ to be only $16 \%$ at $\left\langle q_{z}\right\rangle$ $=0.2 \AA^{-1}$ and much less for higher $\left\langle q_{z}\right\rangle$. If $\left\langle h^{2}\right\rangle \neq 0$, as is most likely to be the case for $-\mathrm{O}-\mathrm{C} 16$ chains, the falloff along $q_{z}$ would be even faster since in that case $S_{\text {rod }}\left(q_{z}\right)$ would acquire a multiplicative Debye-Waller-like factor $\exp \left(-\left\langle h^{2}\right\rangle q_{z}^{2}\right)$. These estimates show that the absence of a measurable peak intensity above $\left\langle q_{z}\right\rangle \sim 0.2 \AA^{-1}$ is roughly consistent with the length of the $-\mathrm{O}-\mathrm{C} 16$ chain.

Having established the peak positions in the reciprocal space, possible packing structures of $-\mathrm{O}-\mathrm{C} 16$ chains can now be considered. The observation of the second peak centered at $q_{x y}=q_{2} \sim 1.68 \AA^{-1}$ and $q_{z}=0$ is significant in that the CS and $L_{2}^{\prime \prime}$ phases of fatty acid, ${ }^{50-53}$ alcohol, ${ }^{50,54}$ and ester $^{34}$ monolayers display a peak at exactly the same location. Of these two low-temperature 2D crystalline phases of single-chain amphiphiles, the CS phase occurs at higher $\Pi$ and consists of long alkyl tails oriented normal to the surface, while the $L_{2}^{\prime \prime}$ phase is a low- $\Pi$ analogue in which tails are tilted towards the nearest neighbors $(\mathrm{NN}){ }^{28}$ Durbin $e t$ al. recently confirmed experimentally that the CS and $L_{2}^{\prime \prime}$ phases, which are related by a continuous transition, arise from a common local packing structure that is based on the herringbone (HB) arrangement of tails. ${ }^{53}$

The HB packing of alkyl chains, which occurs also in bulk organic crystals, has been described previously. ${ }^{28,30,31}$ It is characterized by an orthorhombic (or "distorted hexagon") unit cell of fixed dimensions $a_{1} \times a_{2}=5.0 \AA \times 7.5 \AA$ in the plane perpendicular to chain axes. ${ }^{28,30}$ An end-on view of HB-packed alkyl chains and the rectangular HB unit cell (nonprimitive, two chains per cell) is depicted in Fig. 14(b). Defining 2D reciprocal vectors $G_{h k} \equiv 2 \pi / d_{h k}$ in the plane of the HB unit cell, it can be shown that the two lowest-order sets of reciprocal points correspond to: (i) $G_{11}=1.51 \AA^{-1}$ from the (11) [and $(1 \overline{1})$ ] planes with $d$ spacing $d_{11}$ $=4.16 \AA$, and (ii) $G_{02}=1.68 \AA^{-1}$ from the (02) planes with $d_{02}=3.75 \AA$. That is, if HB-packed alkyl chains are oriented normal to the water surface (as in the CS phase), the (11) and (02) peaks would be centered at $q_{x y}=G_{11}$ and $q_{x y}=G_{02}$ in the surface plane $\left(q_{z}=0\right)$. If the chains that are tilted towards $\mathrm{NN}$ in the [10] direction (as in the $L_{2}^{\prime \prime}$ phase) by angle $\theta$ relative to the surface normal, the (02) peak would still be centered at $q_{z}=0$ since the tilt is perpendicular to the [01] direction; however, the (11) peak would be both shifted inward $\left(q_{x y}<G_{11}\right)$ and lifted above the surface plane $\left(q_{z}\right.$ $>0$ ) such that it falls on the arc $q=\left[q_{x y}^{2}+q_{z}^{2}\right]^{1 / 2}=G_{11}$ at $q_{z}=G_{10} \sin (\theta)$, where $G_{10}=1.26 \AA^{-1} .^{28}$

It is clear from these expectations that the two peaks observed at high $\Pi$ can be identified as the (11) and (02) peaks that originate from the local $\mathrm{HB}$ packing of $-\mathrm{O}-\mathrm{C} 16$ chains. One important observation here is that the HB order of $-\mathrm{O}-\mathrm{C} 16$ chains appears to be a local effect in that the magnitude of the NN tilt is not uniform over the entire area of the C16-O-PBLG monolayer. The appearance of the (11) peak near the surface horizon $\left(q_{z}<0.11 \AA^{-1}\right.$; Fig. 13) indicates that some HB-packed chains must be untilted at high $\Pi$. On the other hand, its shifting behavior $\left(q=G_{11}\right)$ above the surface plane $\left(q_{z}>0.11 \AA^{-1}\right.$; Fig. 15) signifies not only a finite NN tilt of other HB-packed chains but also a continuous distribution in the values of the NN tilt angle $\theta$ that occur 
simultaneously over the surface. For example, at $\Pi=24.0$ dyne/cm, where the (11) peak is still visible at $\left\langle q_{z}\right\rangle_{\max }$ $=0.64 \AA^{-1}$, the tilt angle can be estimated to range from $\theta=0$ (untilted) to more than $\theta=\sin ^{-1}\left(\left\langle q_{z}\right\rangle_{\max } / G_{10}\right) \sim 30^{\circ}$.

The observation of a relatively wide range of tilt angles within a single monolayer is not too surprising for $-\mathrm{O}-\mathrm{C} 16$ chains since they are not isolated molecules sitting on a flat surface but are attached indirectly to the helical backbone. The occurrence of somewhat large tilt angles $\left(\theta \sim 30^{\circ}\right)$ even at high $\Pi$ has already been suggested in the XR section and is roughly consistent with the estimate based on the magnitude of the upper sublayer thickness $l_{\text {chain }}$ (see Sec. III B). Another check on the possibility of large $\theta$ at high $\Pi$ is provided by a comparison between the cross-sectional area $A_{\mathrm{HB}}=a_{1} a_{2} / 2=18.8 \AA^{2} /$ chain taken up by one $-\mathrm{O}-\mathrm{C} 16$ chain in the HB unit cell [Fig. 14(b)] and the area/monomer $A$, which corresponds to the average surface area available per chain. For example, an NN tilt by $\theta=30^{\circ}$ would require an area of $A>A_{\mathrm{HB}} / \cos \left(30^{\circ}\right)=21.7 \AA^{2} /$ monomer, a condition well satisfied up to $\Pi \sim 30$ dyne/cm [Fig. 2(c)].

The extent of lateral correlations associated with the HB order of $-\mathrm{O}-\mathrm{C} 16$ chains can be inferred from the observed peak widths. First, the lack of a fixed NN tilt at given $\Pi$ suggests that the correlations along any in-plane direction with a nonzero component along the NN tilt are likely to be short range. This is consistent with the broadness of the (11) peak in Fig. 13. On the other hand, for highly compressed films, the (11) peak is well defined and its FWHM $\Delta q_{x y}$ $=\Delta q_{1}$ based on the Lorentzian fits can be used to estimate a characteristic correlation length $\xi_{11}=2 /\left[\Delta q_{1}-\delta q_{x y}\right]$ along the direction of $d_{11}$. The correlation length $\xi_{11}$ thus determined is only of the order of $\xi_{11}=13-17 \AA\left(\xi_{11} / d_{11}\right.$ $=3-4$ ) for the PD325 films at $\Pi>25$ dyne/cm and $\xi_{11}$ $=21-25 \AA\left(\xi_{11} / d_{11}=5-6\right)$ for the MD76 films at $\Pi>15$ dyne/cm; it should be even shorter at lower $\Pi$, where the (11) peak is both broader and weaker.

Second, although the amplitude of the (02) peak at $q_{x y}$ $=q_{2}$ is relatively small and the limited number of data points over the peak prevents an accurate determination of its FWHM $\Delta q_{2}$, the width $\Delta q_{2}$ is usually much smaller than $\Delta q_{1}$, and therefore the correlation length $\xi_{02}$ along the [01] axis should be significantly larger than $\xi_{11}$. For example, the fit to the bottommost data curve shown in Fig. 15(a) gives $\Delta q_{2}=0.04 \pm 0.01 \AA^{-1}$, which translates into $\xi_{02}=140$ $\pm 100 \AA$. Taking this as a rough estimate, we expect the correlation length $\xi_{02}$ to be of the order of $\xi_{02} \sim 100 \AA$ or possibly longer. The directional dependence displayed by the relative magnitudes of $\xi_{h k}$ as evidenced by $\xi_{02} \gg \xi_{11}$ is consistent with the case of tilted monolayer phases of alkyl chains, for which the longest positional correlations usually occur along the direction perpendicular to the tilt. ${ }^{28}$

The magnitudes of the correlation lengths $\xi_{h k}$ estimated above suggest that the extent of the HB order of $-\mathrm{O}-\mathrm{C} 16$ chains is actually limited by the physical dimensions of C16-O-PBLG molecules. First of all, $\xi_{02} \sim 100 \AA$ is roughly of the same order of magnitude as the lengths $L$ of typical C16-O-PBLG rods. Second, if the projection $\zeta_{10}$ of $\xi_{11}$ onto the [10] axis (i.e., $\zeta_{10}=a_{2} /\left[a_{1}^{2}+a_{2}^{2}\right]^{1 / 2} \times \xi_{11}$ $\left.=0.83 \xi_{11}\right)$ is taken to be a measure of the HB correlations

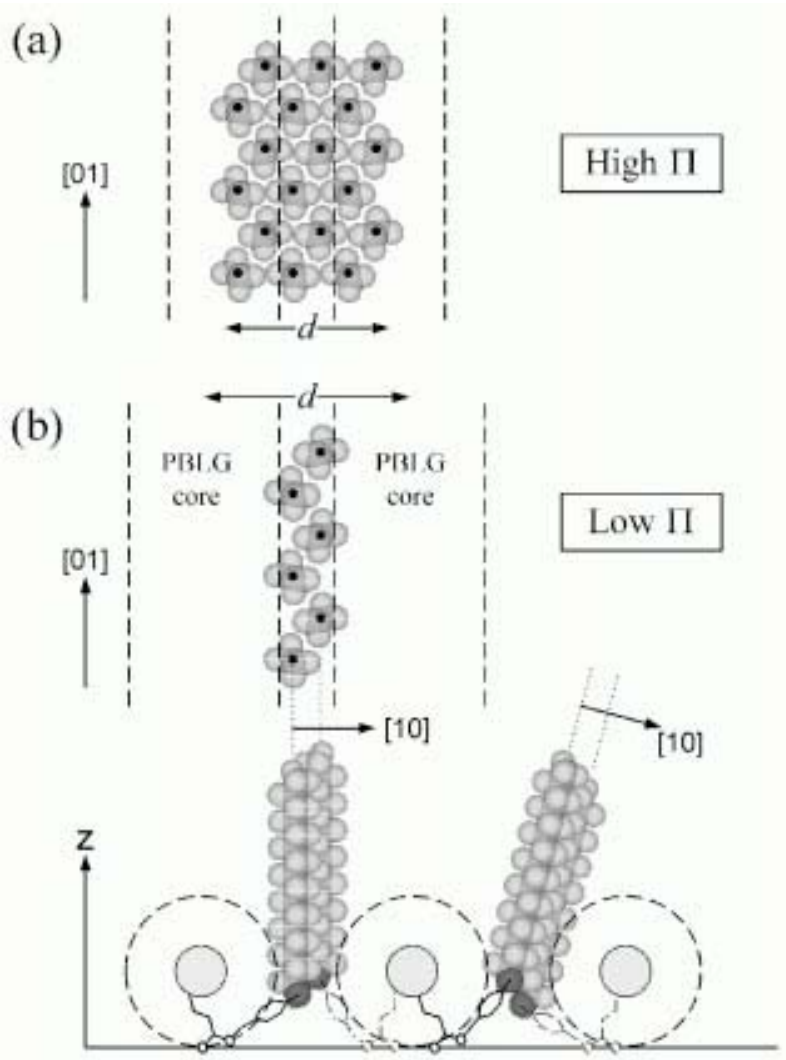

FIG. 16. A model for ordering of side chains, showing ordered chains only. (a) Top view of HB packing at high surface pressure П. The [01] axis of the HB unit cell is oriented parallel to the $\alpha$-helical axes of aligned PBLG cores. (b) Top view and side view of zigzag packing at low $\Pi$, with $1 \mathrm{D}$ HB order for $-\mathrm{O}-\mathrm{C} 16$ chains that are confined between aligned PBLG cores. The $-\mathrm{O}-\mathrm{C} 16$ chains can be untilted or tilted perpendicular to the $\alpha$-helix axes of PBLG cores. In (b), unconfined chains directly above the cores are omitted.

along the $\mathrm{NN}$ tilt direction, then, the maximum value that it reaches upon compression to high $\Pi$ is given by $\zeta_{10, \max }$ $=11-14 \AA$ for the PD325 films and $\zeta_{10, \max }=17-21 \AA$ for the MD76 films. These estimates for $\zeta_{10, \max }$ are comparable to the interhelix distance $d$ observed at high $\Pi$ (see Fig. 12). These observations strongly suggest that the [01] axis of the HB unit cell runs parallel to the helical axes of aligned PBLG cores while the [10] axis and hence the NN tilt of $-\mathrm{O}-\mathrm{C} 16$ chains are in the direction normal to the helical axis.

The well-defined orientation of the HB unit cell with respect to the molecular axis of C16-O-PBLG implies that the HB order must develop with compression in such a way that it is structurally consistent not only with the segregation behavior along the surface normal but also with the in-plane structure of PBLG cores. Figure 16 illustrates one possible model for the spatial development of the HB order that takes into account these various structural aspects of the C16-O-PBLG monolayer. The top view of the monolayer plane in Fig. 16(a) depicts a model structure at high $\Pi$, showing a HB-packed domain of -O-C16 chains with its [01] axis oriented parallel to the lengths of aligned $\mathrm{C} 16-\mathrm{O}_{-}$ PBLG rods. The following considers how such a structure can result from lateral compression of a less twodimensionally ordered structure that is first formed at low $\Pi$. 
The presence of a weak (02) peak and the near absence of the (11) peak at low $\Pi(<\sim 5$ dyne/cm) indicate that a small fraction of the $-\mathrm{O}-\mathrm{C} 16$ chains first form a locally HB-packed structure with a relatively large correlation length parallel to the aligned PBLG-core axes (i.e., along [01]) but with only very limited extent perpendicular to them. The two important characteristics of this initial structure are its pseudo-1D nature and highly dense packing implied by the HB order. If it weren't for the presence of PBLG cores, the latter would be somewhat surprising given the fact that for $\Pi<\sim 5$ dyne $/ \mathrm{cm}$, the average surface area available per chain $A>A_{\lim } \sim 27 \AA^{2} /$ monomer is still significantly larger than $A_{\mathrm{HB}}=18.8 \AA^{2} /$ chain. On the other hand, it has been shown that PBLG cores align locally on the surface without external pressure. Presumably, this reflects a strong mutual attraction and tendency to self-aggregate into a $2 \mathrm{D}$ solid $^{24}$ (see Sec. IIIC1). There are a fixed number of $-\mathrm{O}-\mathrm{C} 16$ chains per unit length of a PBLG core (i.e., $1 / L_{1}$ $=1$ monomer per $1.5 \AA$ ), and approximately one quarter of them are tethered to the water side of the core. Since these chains are hydrophobic, they will have to fold around the subsurface of the core to get away from water. These chains that are confined semi-one-dimensionally between pairs of aligned PBLG cores must then pack more densely than unconfined chains sitting directly above the cores. These confined chains would be subjected to a rather high internal local pressure even at $\Pi=0$. On the basis of these considerations, we suggest that the initial ordering of $-\mathrm{O}-\mathrm{C} 16$ chains at low $\Pi$ is a consequence of the 1D confinement imposed by the local alignments of PBLG cores and can be attributed mostly to chains in the confined regions.

Figure 16(b) illustrates an idealized model (a "zigzag" model) of a possible initial structure $(\Pi<\sim 5$ dyne $/ \mathrm{cm})$ that is based on the HB packing of confined -O-C16 chains. The figure omits unconfined chains, which are expected to be disordered at low $\Pi$. The model consists of two rows of chains forming a zigzag pattern in the confined region, where each row is contributed by one of the two neighboring C16O-PBLG rods on either side. The HB packing implies that the positions of chains along each row are periodic with repeat distance of $a_{2}$ and are related to those of the other row by a vector $\left(a_{1} / 2, a_{2} / 2=d_{02}\right)$ that is normal to the axes of the $-\mathrm{O}-\mathrm{C} 16$ chains. Due to the physical size of alkyl chains, it is not possible to fit more than two rows within the measured gap between the PBLG cores, whose estimated width $\Delta d$ $\sim 5 \AA$ is comparable to $a_{1}$. Given that the side chain as a whole is tethered to the helical backbone, there may be a distribution in the heights of $-\mathrm{O}-\mathrm{C} 16$ chains within a given ordered domain, and any given domain may be tilted in the direction normal to the helical axis [see Fig. 16(b)].

The case for the above interpretation is strongly supported by the following. First, the HB unit cell dimension of $a_{2}=7.5 \AA$ along the [01] axis happens to be an integer multiple of the $\alpha$-helix pitch $L_{1}=1.5 \AA /$ monomer along the helical axis, i.e., $a_{2}=5 L_{1}$. According to the model, on average, every segment of five monomers in a C16-O-PBLG rod would contribute one chain to the zigzag structure on one side of the core and another to the other side. The surface area $A_{5}$ predicted to be taken up by a five-monomer segment of a C16-O-PBLG rod should roughly be equal to $A_{5}$ $\sim 5 A_{\mathrm{PBLG}}+2 A_{\mathrm{HB}}$. Taking the low- $\Pi$ value of $A_{\mathrm{PBLG}}$ $\sim 20 \AA^{2} /$ monomer for the core [Fig. 2(c)], the area/monomer for C16-O-PBLG based on the zigzag model is estimated to be $A_{5} / 5 \sim 27.5 \AA^{2} /$ monomer. The fact that this estimate agrees well with $A_{\text {lim }} \sim 27 \AA^{2} /$ monomer from the isotherms is further evidence for the plausibility of the model. As for the unconfined chains left above the core (roughly three per 5 monomer segment), an average area of (5/3) $A_{\text {PBLG }}$ $\sim 33 \AA^{2} /$ chain available to each at low $\Pi$ is more than enough room for these chains to be disordered.

It should be emphasized that the interpretation given above does not imply that every fifth monomer in a C16-O-PBLG rod contributes its chain to formation of one row in a zigzag structure. In a given $\alpha$ helix, the position at which the side chain of the $m$ th monomer is tethered to the helical backbone can be described by the cylindrical coordinates $\left(r, m \varphi_{1}, m L_{1}\right)$, where $r=2.3 \AA$ is the backbone radius of the $\alpha$ helix and $\varphi_{1}=100^{\circ} .{ }^{55}$ The notion that every fifth monomer has its side chain protruding always on the same side of the rod is inconsistent with the azimuthal angle change of $\Delta \varphi=140^{\circ}$ over five monomers. Given the hydrophobicity of alkyl chains, it is more likely that the monomers contributing to the HB packing in the confined region are those whose side chains are tethered near the bottom (i.e., towards water) of the helical backbone. The ordering of their $-\mathrm{O}-\mathrm{C} 16$ part is probably accomplished by means of some appropriate conformational and orientational rearrangements of the "spacer" part [i.e., $-\left(\mathrm{CH}_{2}\right)_{2}-\mathrm{COO}-\mathrm{CH}_{2}-\mathrm{C}_{6} \mathrm{H}_{4}-$; see Fig. 1(a)], whose end-to-end length can stretch out to $\sim 10 \AA$.

Regardless of the specific molecular-level details of how the surface area is reduced with increasing $\Pi$, it is clear that the free area available to $-\mathrm{O}-\mathrm{C} 16$ chains must become reduced. At high $\Pi(>\sim 5$ dyne/cm), the area constraint will enhance the packing order of chains, thereby increasing the number of unconfined chains participating in the HB order [see Fig. 16(a)]. The area reduction might occur through deformation of PBLG cores, as suggested by the slight increase in $l_{\text {core }}$ with compression (Figs. 8 and 9), or alternatively, by either reducing the tilt within the zigzag structures or vertically displacing one row of chains relative to the other row. While the exact nature of the mechanism is not clear, both the behavior $d=A / L_{1}$ (Fig. 12) and the increase in $l_{\text {chain }}$ at high $\Pi$ indicate that the unconfined $-\mathrm{O}-\mathrm{C} 16$ chains in the upper sublayer do experience effects of reduced area as the film is compressed. Therefore, it is reasonable to suppose that compression causes these chains to be brought into alignment with nearby chains and conform to the HB structures initially formed in the confined regions. This interpretation is consistent with the observed behavior of the (11) peak that implies a lateral growth of the HB order with increasing $\Pi$ in the direction perpendicular to the core axes.

As noted earlier, the C16-O-PBLG monolayer is characterized by the fact that it sustains high surface pressures. This stability can now be understood as a consequence of the lateral ordering of $-\mathrm{O}-\mathrm{C} 16$ chains. According to the estimated values of $\zeta_{10, \max }$ that are reached at high $\Pi,-\mathrm{O}-\mathrm{C} 16$ chains in the MD76 films achieve a slightly higher degree of 
the $\mathrm{HB}$ order perpendicular to PBLG core axes than those in the PD325 films. This is likely to be the origin of the lower compressibility of the MD76 films, which is evidenced by the high- $\Pi$ behaviors of both their isotherms [Fig. 2(c)] and interhelix $d$ spacing (Fig. 12). It is yet unclear how this discrepancy between the two samples depends on the differences in dispersity and molecular weight.

From the structural point of view, the ordering of $-\mathrm{O}-\mathrm{C} 16$ chains evidenced by the results presented above is quite consistent with a highly dense structure based on the HB packing. However, at first glance, the observation of the $\mathrm{HB}$ order at room temperature appears to be in conflict with known thermodynamics of various monolayers formed by simple isolated alkyl chains of comparable length. For example, according to the generalized phase diagram of fatty acid monolayers, the ordered phase formed by $\mathrm{C} 16$ acid at room temperature (and high $\Pi$ ) should have a less dense structure with a hexagonal symmetry, and the $L_{2}{ }^{\prime \prime}$ and CS phases would not occur at all unless the subphase temperature could be reduced to $T<-20{ }^{\circ} \mathrm{C} .{ }^{28}$ However, this discrepancy is not so unreasonable if one takes into account the fact that the $-\mathrm{O}-\mathrm{C} 16$ chain is not an isolated chain but one end of it is attached to a rod-like PBLG core. The constraint of a fixed number of side chains per unit length of $\alpha$ helix together with confinement imposed by the parallel alignments of PBLG cores significantly reduces the number of various degrees of freedom (conformational, rotational, orientational, translational, etc.) that is available to the confined chains. This reduction in chain entropy is probably the reason why $-\mathrm{O}-\mathrm{C} 16$ chains favor the HB structure, which is a well-known low-energy packing mode of alkyl chains. ${ }^{28,30}$

\section{SUMMARY}

Langmuir monolayers of hairy-rod polypeptide C16-O-PBLG have been studied. The $\Pi-A$ isotherms show that the $\mathrm{C} 16-\mathrm{O}-\mathrm{PBLG}$ monolayers sustain much higher surface pressure (up to $\Pi>40$ dyne $/ \mathrm{cm}$ at $22{ }^{\circ} \mathrm{C}$ ) than the PBLG monolayer, which collapses at $\sim 9$ dyne $/ \mathrm{cm}$ to form a bilayer. For $T \geqslant 22^{\circ} \mathrm{C}$, the isotherms of $\mathrm{C} 16-\mathrm{O}-\mathrm{PBLG}$ display a relatively narrow plateau-like feature at low $\Pi(\Delta A / A$ $\sim 15 \%$ or less), while such a feature is absent at $T=11^{\circ} \mathrm{C}$. The exact origin of this low- $\Pi$ behavior is still unknown. However, the reversibility of the plateau feature upon compression and expansion suggests that it is an intrinsic property of the C16-O-PBLG monolayer.

The microscopic structures of the monolayers at room temperature have been probed using $\mathrm{x}$-ray reflectivity and grazing incidence diffraction techniques. The main results can be summarized as follows:

The electron density profiles extracted from XR data are consistent with the formation of a monolayer in which C16-O-PBLG rods are oriented parallel to the water. However, the nonuniformity of the profiles across the film indicates that the hydrophobicity of $-\mathrm{O}-\mathrm{C} 16$ chains results in internal segregation of the monolayer into an upper sublayer occupied by $-\mathrm{O}-\mathrm{C} 16$ chains and a PBLG core-dominated lower sublayer. The monolayer thickens with increasing $\Pi$, but this segregated structure is maintained up to high $\Pi$ ( $\sim 30$ dyne $/ \mathrm{cm})$.
The observation of a low- $q_{x y}$ GID peak at $\Pi \sim 0$ indicates that $\mathrm{C} 16-\mathrm{O}-\mathrm{PBLG}$ rods aggregate laterally and form locally aligned domains in the monolayer. The interhelix distance $d$ between aligned rods decreases continuously with increasing $\Pi$ and scales linearly with $A$ at high $\Pi$. This linear behavior shows that macroscopic compressibility of the monolayer at high $\Pi$ is directly related to the microscopic interhelix compressibility. Due to the presence of $-\mathrm{O}-\mathrm{C} 16$ chains that are confined between aligned PBLG cores, the interhelix $d$ spacing for C16-O-PBLG is larger than that found in the PBLG monolayer. The width $\Delta d \sim 5 \AA$ of this extra spacing for C16-O-PBLG is consistent with the "confined" chains pointing away from water. As in the case of the PBLG monolayer, the observed width of the interhelix peak implies short-range order and the lateral positional correlations between aligned $\mathrm{C} 16-\mathrm{O}-\mathrm{PBLG}$ rods extend over only 2-10 interhelix distances.

For $\Pi>\sim 5$ dyne/cm, GID patterns exhibit two additional peaks at higher $q_{x y}$ that are consistent with ordered packing of $-\mathrm{O}-\mathrm{C} 16$ chains. This suggests that the stability of the C16-O-PBLG monolayers at high $\Pi$ might be a result of the lateral ordering of $-\mathrm{O}-\mathrm{C} 16$ chains in the upper sublayer. The peak positions are consistent with the HB packing of alkyl chains that are commonly found in two low- $T$ phases CS (untilted) and $L_{2}{ }^{\prime \prime}$ (tilted toward NN) of fatty acid monolayers. The results also show that there is a wide distribution in the NN tilt of $-\mathrm{O}-\mathrm{C} 16$ chains, such that the tilt angle for a given HB-packed domain can be anywhere from $0^{\circ}$ (untilted) to $>30^{\circ}$ relative to the surface normal. Various features of these peaks suggest that the HB structure of $-\mathrm{O}-\mathrm{C} 16$ chains has a specific in-plane orientation with respect to the helical axes of aligned PBLG cores. The orientation is such that the NN tilt direction is always perpendicular to the helix axes.

For $\Pi<\sim 5$ dyne/cm, one of the two peaks is absent. This observation together with the results at high $\Pi$ suggests that the initial structure formed at low $\Pi$ has onedimensional character, such that the extent of HB order is relatively large only in the direction parallel to the axes of aligned PBLG cores. We have proposed a model in which the initial one-dimensionally ordered structure consists of $\mathrm{HB}$ packing of "confined" $-\mathrm{O}-\mathrm{C} 16$ chains. The model is consistent with the various structural characteristics of the monolayers that have been elucidated in this study. In the C16-O-PBLG monolayers, the HB order of $-\mathrm{O}-\mathrm{C} 16$ chains appears to be a consequence of the $1 \mathrm{D}$ confinement imposed by the local alignments of PBLG cores and the tethering constraints and hydrophobicity of these chains.

\section{ACKNOWLEDGMENTS}

The Harvard contribution to this work was supported by Grant No. NSF-DMR-01-24936. D.T. acknowledges support by Grant No. NSF-DMR-01-10437. NSLS at Brookhaven National Laboratory is supported by Grant No. DE-AC02$76 \mathrm{CH} 00016$. 
${ }^{1}$ G. Wegner, Thin Solid Films 216, 105 (1992).

${ }^{2}$ G. Wegner and K. Mathauer, Mater. Res. Soc. Symp. Proc. 247, 767 (1992).

${ }^{3}$ J. Watanabe, Y. Fukuda, R. Gehani, and I. Uematsu, Macromolecules 17, 1004 (1984).

${ }^{4}$ J. Watanabe, H. Ono, I. Uematsu, and A. Abe, Macromolecules 18, 2141 (1985).

${ }^{5}$ E. Iizuka, K. Abe, K. Hanabusa, and H. Shirai, Current Topics in Polymer Science (Hanser, Munich, 1987), Vol. I, p. 235.

${ }^{6}$ K. Hanabusa, M. Sato, H. Shirai, K. Takemoto, and E. Iizuka, J. Polym. Sci., Polym. Lett. Ed. 22, 559 (1984).

${ }^{7}$ S. M. Yu and D. A. Tirrell, Biomacromolecules 1, 310 (2000).

${ }^{8}$ H. Motschmann, R. Reiter, R. Lawall, G. Duda, M. Stamm, G. Wegner, and W. Knoll, Langmuir 7, 2743 (1991).

${ }^{9}$ D. Sohn, H. Yu, J. Nakamatsu, P. S. Russo, and W. H. Daly, J. Polym. Sci. [B] 34, 3025 (1996).

${ }^{10}$ D. Neher, Adv. Mater. (Weinheim, Ger.) 7, 691 (1995).

${ }^{11}$ W. Hickel, G. Duda, M. Jurich, T. Krohl, K. Rochford, G. I. Stegeman, J. D. Swalen, G. Wegner, and W. Knoll, Langmuir 6, 1403 (1990).

${ }^{12}$ K. Mathauer, A. Mathy, C. Bubeck, G. Wegner, W. Hickel, and U. Scheunemann, Thin Solid Films 210/211, 449 (1992).

${ }^{13}$ A. Mathy, K. Mathauer, G. Wegner, and C. Bubeck, Thin Solid Films 215, 98 (1992).

${ }^{14}$ H. Menzel, B. Weichart, and M. L. Hallensleben, Thin Solid Films 223, 181 (1993).

${ }^{15}$ H. Menzel, M. L. Hallensleben, A. Schmidt, W. Knoll, T. Fischer, and J. Stumpe, Macromolecules 26, 3644 (1993).

${ }^{16}$ M. Mabuchi, S. Ito, M. Yanamoto, T. Miyamoto, A. Schmidt, and W. Knoll, Macromolecules 31, 8802 (1998).

${ }^{17}$ A. Vogel and B. Hoffmann, Sens. Actuators B 4, 65 (1991).

${ }^{18}$ G. Wiegand, T. Jaworek, G. Wegner, and E. Sackmann, Langmuir 13, 3563 (1997).

${ }^{19}$ H. Menzel, B. Weichart, and M. L. Hallensleben, Thin Solid Films 242, 56 (1994).

${ }^{20}$ A. Schmidt, K. Mathauer, G. Reiter, M. D. Foster, M. Stamm, G. Wegner, and W. Knoll, Langmuir 10, 3820 (1994).

${ }^{21}$ T. R. Vierheller, M. D. Foster, A. Schmidt, K. Mathauer, W. Knoll, G. Wegner, S. Satija, and C. F. Majkrzak, Macromolecules 27, 6893 (1994).

${ }^{22}$ K. Mathauer, A. Schmidt, W. Knoll, and G. Wegner, Macromolecules 28, 1214 (1995).

${ }^{23}$ M. Buchel, Z. Sekkat, S. Paul, B. T. Weichart, H. Menzel, and W. Knoll, Langmuir 11, 4460 (1995).

${ }^{24}$ M. Fukuto, R. K. Heilmann, P. S. Pershan, S. M. Yu, J. A. Griffiths, and D. A. Tirrell, J. Chem. Phys. 111, 9761 (1999).

${ }^{25}$ C. M. Knobler, Adv. Chem. Phys. 77, 397 (1990).

${ }^{26}$ C. M. Knobler and R. C. Desai, Annu. Rev. Phys. Chem. 43, 207 (1992).

${ }^{27}$ H. Möhwald, Annu. Rev. Phys. Chem. 41, 441 (1990).

${ }^{28}$ V. M. Kaganer, H. Möwald, and P. Dutta, Rev. Mod. Phys. 71, 779 (1999).

${ }^{29}$ M. Fukuto, R. K. Heilmann, P. S. Pershan, S. M. Yu, C. M. Soto, and D. A. Tirrell, Phys. Rev. E 66, 010601(R) (2002).
${ }^{30}$ I. Kuzmenko, V. M. Kaganer, and L. Leiserowitz, Langmuir 14, 3882 (1998).

${ }^{31}$ D. M. Small, The Physical Chemistry of Lipids: From Alkanes to Phospholipids (Plenum, New York, 1986).

${ }^{32}$ S. M. Yu, V. Conticello, G. Zhang, C. Kayser, M. J. Fournier, T. L. Mason, and D. A. Tirrell, Nature (London) 389, 167 (1997).

${ }^{33}$ D. K. Schwartz, M. L. Schlossman, and P. S. Pershan, J. Chem. Phys. 96, 2356 (1992)

${ }^{34}$ W. J. Foster, M. C. Shih, and P. S. Pershan, J. Chem. Phys. 105, 3307 (1996).

${ }^{35}$ G. M. Bommarito, W. J. Foster, P. S. Pershan, and M. L. Schlossman, J. Chem. Phys. 105, 5265 (1996).

${ }^{36} \mathrm{~J}$. Als-Nielsen and H. Möhwald, in Handbook of Synchrotron Radiation, edited by S. Ebashi, E. Rubenstein, and M. Koch (North-Holland, Amsterdam, 1991), Vol. 4, p. 1.

${ }^{37}$ J. Als-Nielsen, D. Jacquemain, K. Kjaer, F. Leveiller, M. Lahav, and L. Leiserowitz, Phys. Rep. 246, 251 (1994).

${ }^{38}$ P. S. Pershan and J. Als-Nielsen, Phys. Rev. Lett. 52, 759 (1984).

${ }^{39}$ B. M. Ocko, X. Z. Wu, E. B. Sirota, S. K. Sinha, O. Gang, and M. Deutsch, Phys. Rev. E 55, 3164 (1997).

${ }^{40}$ B. G. Moore, C. M. Knobler, S. Akamatsu, and F. Rondelez, J. Phys. Chem. 94, 4588 (1990).

${ }^{41}$ I. R. Peterson, V. Brzezinski, R. M. Kenn, and R. Steitz, Langmuir 8, 2995 (1992).

${ }^{42}$ G. A. Overbeck and D. Mobius, J. Phys. Chem. 97, 7999 (1993).

${ }^{43}$ A. Braslau, P. S. Pershan, G. Swislow, B. M. Ocko, and J. Als-Nielsen, Phys. Rev. A 38, 2457 (1988).

${ }^{44}$ D. K. Schwartz, M. L. Schlossman, E. H. Kawamoto, G. J. Kellogg, P. S. Pershan, and B. M. Ocko, Phys. Rev. A 41, 5687 (1990).

${ }^{45}$ I. M. Tidswell, B. M. Ocko, P. S. Pershan, S. R. Wasserman, G. M. Whitesides, and J. D. Axe, Phys. Rev. B 41, 1111 (1990).

${ }^{46}$ X. Z. Wu, E. B. Sirota, S. K. Sinha, B. M. Ocko, and M. Deutsch, Phys. Rev. Lett. 70, 958 (1993).

${ }^{47}$ J. Daillant, L. Bosio, J. J. Benattar, and J. Meunier, Europhys. Lett. 8, 453 (1989).

${ }^{48}$ J. Daillant, L. Bosio, and J. J. Benattar, Europhys. Lett. 12, 715 (1990).

${ }^{49}$ J. P. Rieu, J. F. Legrand, A. Renault, B. Berge, B. M. Ocko, X. Z. Wu, and M. Deutsch, J. Phys. II 5, 607 (1995).

${ }^{50}$ T. M. Bohanon, B. Lin, M. C. Shih, G. E. Ice, and P. Dutta, Phys. Rev. B 41, 4846 (1990).

${ }^{51}$ B. Lin, M. C. Shih, T. M. Bohanon, G. E. Ice, and P. Dutta, Phys. Rev. Lett. 65, 191 (1990).

${ }^{52}$ R. M. Kenn, C. Böhm, A. M. Bibo, I. R. Peterson, H. Möhwald, K. Kjær, and J. Als-Nielsen, J. Phys. Chem. 95, 2092 (1991).

${ }^{53}$ M. K. Durbin, A. G. Richter, C.-J. Yu, J. Kmetko, J. M. Bai, and P. Dutta, Phys. Rev. E 58, 7686 (1998).

${ }^{54}$ M. C. Shih, T. M. Bohanon, J. M. Mikrut, P. Zschack, and P. Dutta, J. Chem. Phys. 97, 4485 (1992).

${ }^{55}$ G. E. Schulz and R. H. Schirmer, Principles of Protein Structure (Springer, New York, 1979). 